\title{
Evidence-based Clinical Decision Support Systems for the \\ prediction and detection of three disease states in critical
}

\section{care: A systematic literature review [version 1; peer review: 1}

\section{approved, 1 approved with reservations]}

\author{
Goran Medic (iD1,2, Melodi Kosaner Kließ33, Louis Atallah4, Jochen Weichert4, \\ Saswat Panda ${ }^{3}$, Maarten Postma2,5,6, Amer EL-Kerdi ${ }^{4}$ \\ ${ }^{1}$ Health Economics, Philips, Eindhoven, Noord-Brabant, 5621JG, The Netherlands \\ ${ }^{2}$ Department of Pharmacy, Unit of PharmacoTherapy, -Epidemiology \& -Economics, University of Groningen, Groningen, 9700 AB, \\ The Netherlands \\ ${ }^{3}$ Global Market Access Solutions Sàrl, St-Prex, 1162, Switzerland \\ ${ }^{4}$ Philips, Cambridge, MA, 02141, USA \\ ${ }^{5}$ Department of Health Sciences, University Medical Centre Groningen, University of Groningen, Groningen, 9700 AB, The \\ Netherlands \\ ${ }^{6}$ Department of Economics, Econometrics \& Finance, University of Groningen, Groningen, 9700 AB, The Netherlands
}

V1 First published: 08 Oct 2019, 8:1728

https://doi.org/10.12688/f1000research.20498.1

Latest published: 27 Nov 2019, 8:1728

https://doi.org/10.12688/f1000research.20498.2

\section{Abstract}

Background: Clinical decision support (CDS) systems have emerged as tools providing intelligent decision making to address challenges of critical care. CDS systems can be based on existing guidelines or best practices; and can also utilize machine learning to provide a diagnosis, recommendation, or therapy course.

Methods: This research aimed to identify evidence-based study designs and outcome measures to determine the clinical effectiveness of clinical decision support systems in the detection and prediction of hemodynamic instability, respiratory distress, and infection within critical care settings. PubMed, ClinicalTrials.gov and Cochrane Database of Systematic Reviews were systematically searched to identify primary research published in English between 2013 and 2018. Studies conducted in the USA, Canada, UK, Germany and France with more than 10 participants per arm were included.

Results: In studies on hemodynamic instability, the prediction and management of septic shock were the most researched topics followed by the early prediction of heart failure. For respiratory distress, the most popular topics were pneumonia detection and prediction followed by pulmonary embolisms. Given the importance of imaging and clinical notes, this area combined Machine Learning with image analysis and natural language processing. In studies on infection, the most researched areas were the detection, prediction,

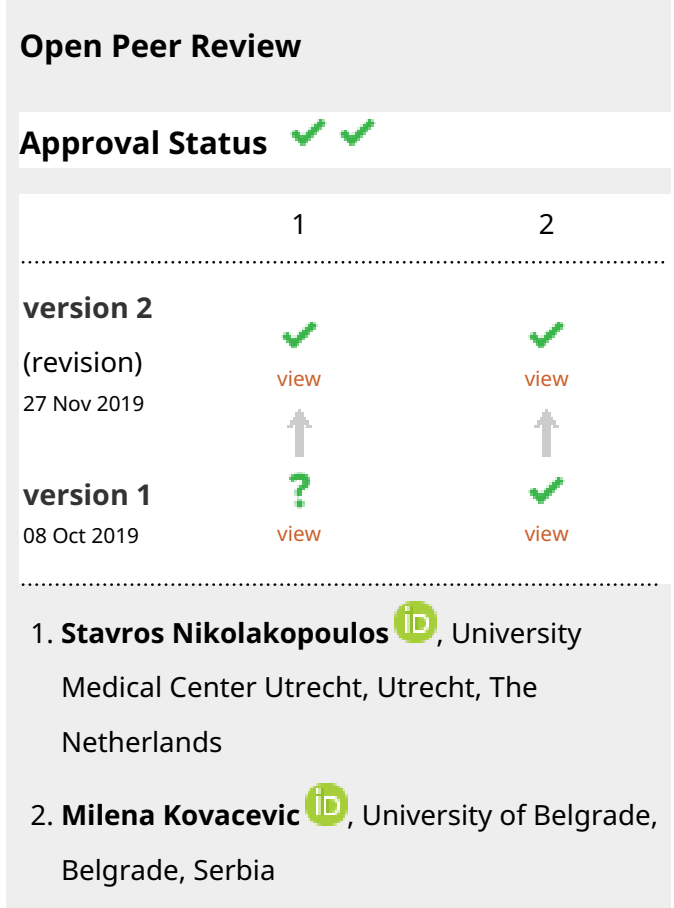

Any reports and responses or comments on the article can be found at the end of the article. 
and management of sepsis, surgical site infections, as well as acute kidney injury. Overall, a variety of Machine Learning algorithms were utilized frequently, particularly support vector machines, boosting techniques, random forest classifiers and neural networks. Sensitivity, specificity, and ROC AUC were the most frequently reported performance measures.

Conclusion: This review showed an increasing use of Machine Learning for CDS in all three areas. Large datasets are required for training these algorithms; making it imperative to appropriately address, challenges such as class imbalance, correct labelling of data and missing data. Recommendations are formulated for the development and successful adoption of CDS systems.

\section{Keywords}

sepsis, hemodynamic instability, respiratory distress, infection, machine learning, clinical trials, critical care.

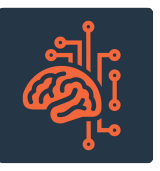

This article is included in the Artificial

Intelligence and Machine Learning gateway.

Corresponding author: Goran Medic (goran.medic@philips.com)

Author roles: Medic G: Conceptualization, Data Curation, Funding Acquisition, Methodology, Project Administration, Supervision, Validation, Writing - Original Draft Preparation; Kosaner Kließ M: Data Curation, Formal Analysis, Methodology, Project Administration, Validation, Writing - Review \& Editing; Atallah L: Writing - Original Draft Preparation, Writing - Review \& Editing; Weichert J: Writing Review \& Editing; Panda S: Data Curation, Formal Analysis, Investigation, Methodology, Validation, Writing - Review \& Editing; Postma M: Conceptualization, Supervision, Writing - Review \& Editing; EL-Kerdi A: Conceptualization, Funding Acquisition, Methodology, Supervision, Validation, Writing - Review \& Editing

Competing interests: PM has no conflicts of interest. MG, AL, WJ and ELKA are the employees of Philips. KKM and PS are the employees of Global Market Access Solutions Sàrl. Global Market Access Solutions Sàrl. Received funding from Philips to perform systematic literature review. PM is the employee of the University of Groningen, The Netherlands who provided scientific oversight for the whole project and did not receive any financial support.

Grant information: The study was supported by funding from Philips.

The funders had no role in study design, data collection and analysis, decision to publish, or preparation of the manuscript.

Copyright: @ 2019 Medic G et al. This is an open access article distributed under the terms of the Creative Commons Attribution License, which permits unrestricted use, distribution, and reproduction in any medium, provided the original work is properly cited.

How to cite this article: Medic G, Kosaner Kließ M, Atallah L et al. Evidence-based Clinical Decision Support Systems for the prediction and detection of three disease states in critical care: A systematic literature review [version 1; peer review: 1 approved, 1 approved with reservations] F1000Research 2019, 8:1728 https://doi.org/10.12688/f1000research.20498.1

First published: 08 Oct 2019, 8:1728 https://doi.org/10.12688/f1000research.20498.1 


\section{Introduction}

Critical care, including intensive and emergency care, is the most expensive and human resource intensive area of in-hospital care. Despite having the most technologically advanced devices, it is the area associated with the highest morbidity and mortality rates ${ }^{1}$. Decision-making for clinical teams in this area is complex due to variability in procedures and dataoverload from the plethora of existing devices. In fact, misdiagnosis in the intensive care unit (ICU) is 50\% more common than other areas $^{2}$, and errors, especially medication errors which account for $78 \%$ of serious medication errors ${ }^{3}$, can have a long lasting effect even after patients are discharged.

Computerized decision support (CDS) systems have emerged as tools providing intelligent decision making based on patient data to address many of the challenges of critical care. CDS systems can be based on existing guidelines or best practices; and can also utilize machine learning as a means of compiling several data inputs to provide a diagnosis, recommendation, or therapy course. CDS systems can improve medication safety by providing recommendations relating to dosing ${ }^{4-6}$, administration frequencies ${ }^{5}$, medication discontinuation ${ }^{6}$ and medication avoidance $^{5}$. Moreover, these novel systems can improve the quality of prescribing decisions by triggering alerts or warning messages on drug duplication, contraindications, drug interaction errors ${ }^{7}$, side-effects and inappropriate medication orders ${ }^{5}$. CDS system notifications can be applied during the prescribing, administering or monitoring stages to detect and prevent medication errors ${ }^{8}$. These systems can also target patients to facilitate shared decision-making to empower as well as to motivate them ${ }^{9-11}$. The need for such systems stems from hospitals having to deal with strict guidelines to improve outcomes, document care cycles (raising the need for administrative tasks) and reduce readmissions. This is combined with the need to cope with financial constraints, such as staff shortages and increased pressure to reduce the length of stay ${ }^{12,13}$.

Strategies for bringing CDS to clinics have been the topic of several workshops, conferences and focus groups ${ }^{14}$. Factors for success in designing CDS include providing measurable value, producing actionable insights, delivering information to the user at the right time, and demonstrating good usability principles ${ }^{14}$.

Early warning systems (EWS) are CDS systems designed for initial assessment and identification of patients at risk of deterioration in in-patient ward areas ${ }^{15-17}$. These systems have shown that they can enable caregivers and rapid response teams to respond earlier - in time to make a difference ${ }^{18}$. By alerting clinicians to higher risk patients, treatments can be administered early or harmful medications can be stopped, potentially leading to improved outcomes. Early recognition and timely intervention are also critical steps for the successful management of shock $^{19}$, cardiorespiratory instability ${ }^{20}$ and severe sepsis. In sepsis management, adequate timing of administration of antibiotics is directly associated with survival rates $^{21}$, and incidence, severity and duration of infections.

According to the Society of Critical Care Medicine (SCCM) ${ }^{22}$, the five primary ICU admission diagnoses for adults are respiratory insufficiency/failure with ventilator support, acute myocardial infarction, intracranial hemorrhage or cerebral infarction, percutaneous cardiovascular procedures, and septicemia or severe sepsis without mechanical ventilation. SCCM also highlights other conditions involving high ICU demand such as poisoning and toxic effects of drugs, pulmonary edema and respiratory failure, heart failure and shock, cardiac arrhythmia and renal failure. Given the above, three high-impact areas were selected for the current research where early detection and treatment could impact outcomes for patients in the ICU. The first is that of hemodynamic instability, where early detection could help patients prevent deterioration into shock. The second is that of respiratory distress, affecting many ventilated patients (up to $40 \%$ are ventilated according to SCCM $)^{22}$. The third area selected is that of infection, with a focus on sepsis. Sepsis is the most common cause of death among critically ill patients, with occurrence rates varying from $13.6 \%$ to $39.3 \%{ }^{23,24}$. All three areas are major areas of concern with relatively high prevalence in critical care having long term effects on patients.

The study focuses on both detection, which alerts the clinician to the presence of these specific conditions, as well as prediction of deterioration by alerting the clinician in advance that a patient will deteriorate into one of these disease states. The aims of this study were to perform and report a systematic review of the utilization of CDS systems in the three selected disease areas and summarize the methodological aspects of identified studies.

\section{Methods}

Search strategy

A systematic literature review was carried out to identify evidence-based study designs, methods and outcome measures that have been used to determine the clinical effectiveness of CDS systems in the detection and prediction of three populations representing the variety and majority of morbid conditions in a critical care setting: Shock (hemodynamic (in-)stability), respiratory distress/failure and infection/sepsis. The search strategy combined 'intervention terms' and 'disease terms' to identify primary research evaluating the diagnostic performance of CDS systems and other machine learning algorithms in three different populations of any age, sex, and race. Systematic literature reviews were also included for locating further relevant primary research. The search was conducted in MEDLINE (PubMed), ClinicalTrials.gov and Cochrane Database of Systematic Reviews (CDSR); and limited to studies published or registered between January 1, 2013 and November 8, 2018 and reported in English. Publication dates were limited to focus results on the most recent developments in this fast-evolving research domain. The strategy employed in PubMed is provided as Extended data, Table 1-Table $3^{25-27}$.

Studies conducted in US, Canada, UK, Germany or France with more than 10 subjects per arm were included. These countries were selected because they are known to be active in CDS development. The inclusion and exclusion criteria for selecting abstracts and subsequent full-text publications were based on the population, interventions, comparators, outcomes, and study design (PICOS). These criteria are listed in Table 1. 
Table 1. Study selection criteria for the systematic literature review.

\begin{tabular}{|c|c|c|c|}
\hline \multicolumn{2}{|l|}{ Criteria } & \multirow[b]{2}{*}{$\begin{array}{l}\text { Inclusion } \\
\text { Randomized controlled trials (RCT) } \\
\text { Observational (retrospective and prospective) } \\
\text { studies } \\
\text { In-hospital settings: Acute care, Intensive care } \\
\text { unit (ICU), Emergency department (ED), Medical } \\
\text { Surgery, General ward } \\
\text { Geography: US, Canada, Europe }\end{array}$} & \multirow[b]{2}{*}{$\begin{array}{l}\text { Exclusion } \\
\text { Systematic Literature Reviews or meta- } \\
\text { analyses* } \\
\text { Review papers, newsletters and opinion } \\
\text { papers where treatments of interest are only } \\
\text { discussed } \\
\text { Methodology studies or protocols } \\
\text { Case studies (sample size of } 1 \text { patient) } \\
\text { Studies with less than } 10 \text { patients per arm; } \\
\text { Conference abstracts published only as } \\
\text { abstracts in } 2013,2014,2015 \text { and } 2016 \\
\text { Geography*: All countries and regions } \\
\text { except: US, Canada, UK, Germany, France } \\
\text { Publications without an abstract }\end{array}$} \\
\hline STUDY DESIGN & $\begin{array}{l}\text { Abstract } \\
\text { selection }\end{array}$ & & \\
\hline & $\begin{array}{l}\text { Full-text } \\
\text { selection }\end{array}$ & $\begin{array}{l}\text { Randomized controlled trials (RCT) } \\
\text { Observational (retrospective and prospective) } \\
\text { studies } \\
\text { In-hospital settings: Acute care, Intensive care } \\
\text { unit (ICU), Emergency department (ED), Medical } \\
\text { Surgery, General ward } \\
\text { Geography }{ }^{\star *} \text { US, Canada, UK, Germany, France } \\
\text { Conference abstracts published only as abstracts in } \\
2017 \text { and } 2018\end{array}$ & $\begin{array}{l}\begin{array}{l}\text { Systematic Literature Reviews or meta- } \\
\text { analyses }\end{array} \\
\text { Review papers, newsletters and opinion } \\
\text { papers where treatments of interest are only } \\
\text { discussed } \\
\text { Methodology studies or protocols } \\
\text { Case studies (sample size of } 1 \text { patient) } \\
\text { Studies with less than } 10 \text { patients per arm; } \\
\text { Geography }{ }^{* *} \text { : All countries and regions } \\
\text { except: US, Canada, UK, Germany, France } \\
\text { Publications published only as abstracts in } \\
2013,2014,2015 \text { and } 2016 \text { (which were not } \\
\text { superseded by full-text publication). }\end{array}$ \\
\hline POPULATION & $\begin{array}{l}\text { Abstract } \\
\text { and full-text } \\
\text { selection }\end{array}$ & $\begin{array}{l}\text { Studies that include humans only -adults, children } \\
\text { and neonates (or (electronic) medical records) } \\
\text { Both sexes are included Patients with or at risk of } \\
\text { developing shock (hemodynamic (in-stability) } \\
\text { Patients with or at risk of developing respiratory } \\
\text { distress/failure } \\
\text { Patients with or at risk of developing infection or } \\
\text { sepsis } \\
\text { Healthy people only; Healthy people and patients }\end{array}$ & $\begin{array}{l}\text { In-vitro studies } \\
\text { Animal studies }\end{array}$ \\
\hline $\begin{array}{l}\text { TREATMENT / } \\
\text { INTERVENTION }\end{array}$ & $\begin{array}{l}\text { Abstract } \\
\text { and full-text } \\
\text { selection }\end{array}$ & $\begin{array}{l}\text { Artificial intelligence } \\
\text { Machine learning e.g. Deep learning models) } \\
\text { Clinical decision support } \\
\text { Computer aided detection } \\
\text { Early Warning System }\end{array}$ & $\begin{array}{l}\text { Automatic diagnosis systems (i.e. ELISA } \\
\text { tests) } \\
\text { Screening tests (i.e. Automated analysis of } \\
\text { portable oximetry) } \\
\text { Sequencing tests } \\
\text { Mathematical models } \\
\text { the predictability of disease or treatment/ } \\
\text { intervention (i.e. Modelling studies have been } \\
\text { widely used to inform human papillomavirus } \\
\text { vaccination policy decisions) } \\
\text { Multivariable hierarchal logistic regression } \\
\text { models } \\
\text { statistics - but there is no machine learning) }\end{array}$ \\
\hline COMPARATOR & $\begin{array}{l}\text { Abstract } \\
\text { and full-text } \\
\text { selection }\end{array}$ & All comparators & $\begin{array}{l}\text { No selection will be made regarding } \\
\text { comparator }\end{array}$ \\
\hline
\end{tabular}




\begin{tabular}{|c|c|c|c|}
\hline Criteria & & Inclusion & Exclusion \\
\hline OUTCOMES & $\begin{array}{l}\text { Abstract } \\
\text { and full-text } \\
\text { selection }\end{array}$ & 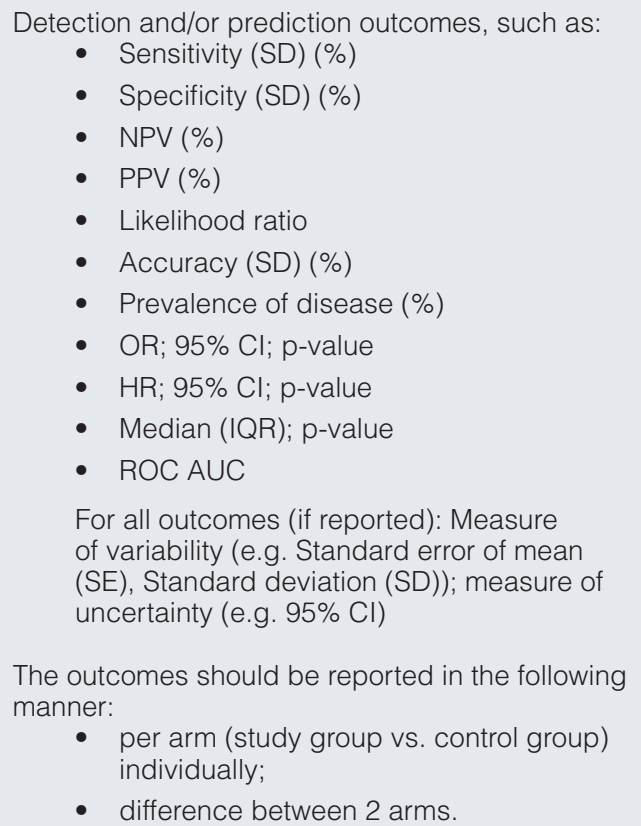 & $\begin{array}{l}\text { Studies not reporting detection and/or } \\
\text { prediction outcomes } \\
\text { Studies discussing interventions of interest, } \\
\text { but no outcomes are reported }\end{array}$ \\
\hline
\end{tabular}

* Systematic Literature Reviews and (network) meta-analysis are excluded from data extraction since the pooled results cannot be used in our analysis. However, good quality (network) meta-analysis and systematic literature reviews (i.e. Cochrane reviews) will be used for cross-checking of references if the search did not omit any articles.

** If studies are conducted in multiple countries and at least 1 of the included countries is included - the study will be included in the selection.

*** Mathematical and logistic regression models - can be used to validate and evaluate Interventions of interest (that are listed as included intervention), but the texts discussing these models without any "learning potential" or artificial intelligence potential will be excluded. Therefore, these models can be the foundation of the included listed interventions but will not be included in the Data Extraction Files unless they have also machine learning or artificial intelligence or some other form of "learning potential" on top of the statistical mathematical model. Researchers will pay special attention and caution when screening these abstracts and/or full-text articles.

AUC = Area under the curve; ED = Emergency department; ELISA = Enzyme-linked immunosorbent assay; HR = Hazard ratio; ICU = Intensive care unit; $\mathrm{IQR}=$ interquartile range; NPV = Negative predictive value; OR = Odds ratio; PPV = Positive predictive value; RCT = Randomized controlled trial; ROC = Receiver Operating Characteristic; SD = Standard deviation; SE = Standard error; UK = United Kingdom; US = United States.

\section{Study selection and data extraction}

Study selection and data extraction was carried out by a single reviewer (MKK or SP). In cases of uncertainty, a second, or even third reviewer, was consulted. Data extraction was performed using a standard data extraction form (DEF). Key data from each additional eligible study were extracted by recording data from original reports into the DEF. The DEF included information on study design, inclusion/exclusion criteria, sample size and characteristics, interventions, outcome measures (measures of predictability like: sensitivity, specificity, negative predictive value (NPV), positive predictive value (PPV), likelihood ratio, accuracy, odds ratio (OR), hazard ratio (HR), median, receiver operating characteristic (ROC) area under the curve (AUC); and length of hospitalization among others).

Studies identified from the ClinicalTrials.gov registry that did not report results were also included in the extraction to give some indication of the outcomes being collected.

\section{Study quality appraisal}

This research was not aimed at summarizing study results and assessing the relative effectiveness of CDS systems. Therefore, an appraisal of study quality was not deemed necessary.

\section{Results}

Shock (hemodynamic (in-)stability)

The search yielded 1588 hits. Screening the titles and abstracts led to 1502 being excluded. The full texts of the remaining 86 titles were obtained and assessed against the PICOS criteria. Studies were excluded due to irrelevant study design $(n=22)$, population $(n=1)$, intervention $(n=5)$, and outcomes $(n=38)$. A total of 20 studies were finally included in this systematic literature review. This included 5 trials identified from ClinicalTrials.gov. The study selection process is depicted in Figure 1.

Study characteristics. Of the 15 published studies, five were conducted by research groups outside the USA ${ }^{28-32}$. Ten studies were conducted in the US ${ }^{19,33-41}$, Thirteen studies were retrospective $\mathrm{i}^{1928-33,35,37-41}$ and only two were prospective ${ }^{34,36}$. Nine studies were single-center ${ }^{28,30,31,33,37-41}$ and six studies were multi-center ${ }^{19,29,32,34-36}$. Five studies were time-series ${ }^{28,30-32,40}$ and nine were case-series ${ }^{19,29,33-35,37-39,41}$.

Across all studies, three had sample sizes $\leq 100^{29,30,36}$; three had sample sizes of 101-100028,31,32; four studies had sample sizes of 1001-10,000 19,33,34,37,42; and another five studies, four retrospective 


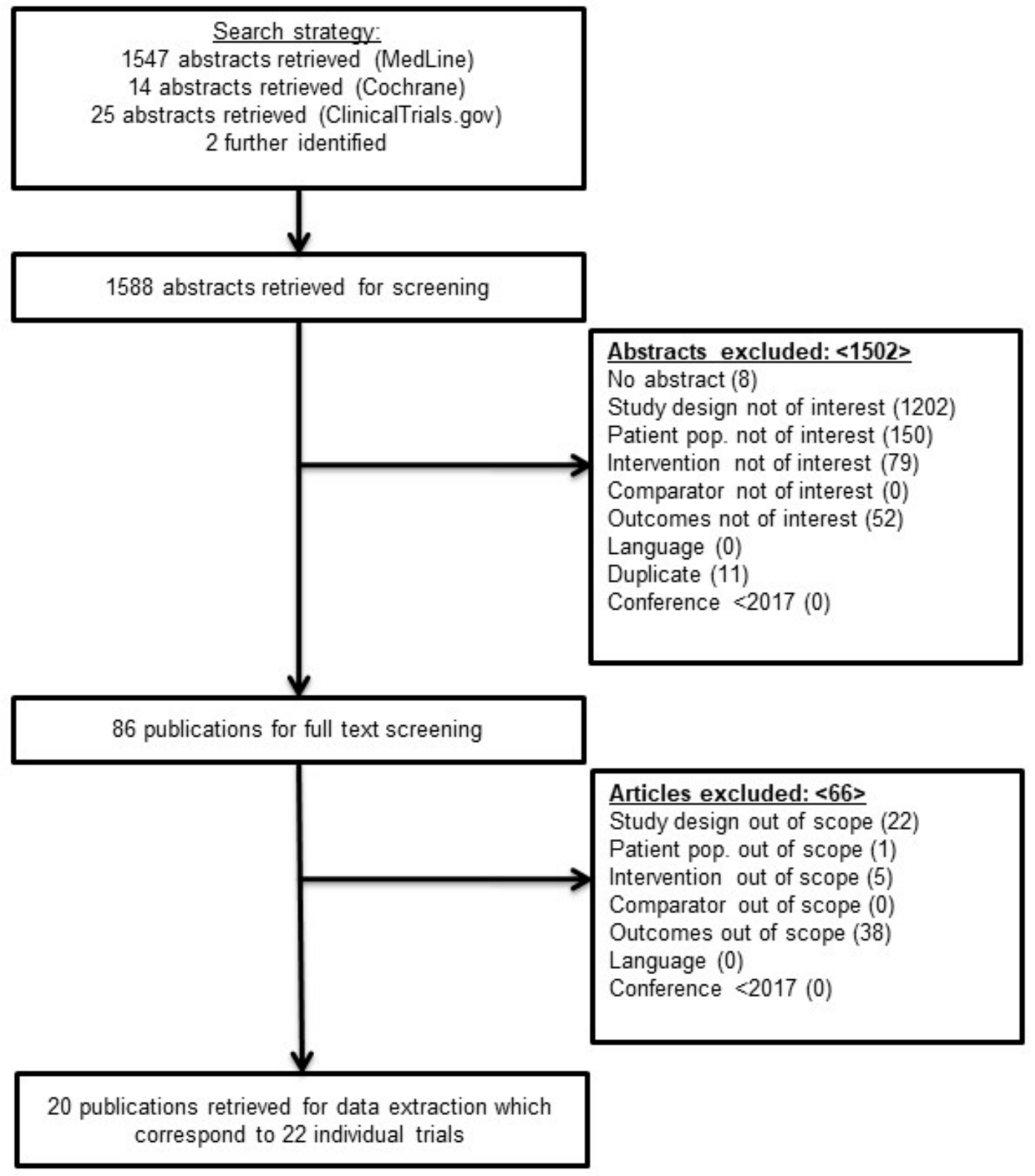

Figure 1. Study selection - Shock. Pop. = Population. 
single-center studies and one multi-center, had sample sizes larger than $10,000^{35,38-41}$. The three largest studies included patients admitted to various wards of a specified hospital. The majority of the studies did not restrict their sample to a specific in-patient hospital setting. Five studies reported on patients in the $\operatorname{ICU}^{19,28,32,40,41}$ and one study reported on patients admitted to the surgical ward ${ }^{33}$.

The characteristics of the published studies are summarized in Table 2.

CDS systems. Machine learning algorithms were developed to detect or predict septic shock ${ }^{28,33,35,40,41}$, various heart $\operatorname{arrhythmias}^{29,30,34}$, heart failure ${ }^{37-39}$, hemodynamic instability and hypovolemia $^{19,36}$, myocardial infarction ${ }^{31}$, as well as hypotension ${ }^{32}$.

All studies, except one, trained a single algorithm. Ebrahimzadeh et al. $2018^{30}$ trained and compared support vector machine (SVM), instance-based and neural network models to predict paroxysmal atrial fibrillation. SVMs were the most frequently used algorithms, followed by least absolute shrinkage and selection operator (LASSO) regularization. In one study, the SVM was trained using sequential minimal optimization ${ }^{37}$. An overview of the investigated machine learning algorithms is presented in Table 3.

Outcome measures. Three of the 15 papers measured a single outcome of model performance. In two studies the preferred measure was accuracy ${ }^{28,34}$; whereas in another study this was the ROC AUC. This study was large and based their algorithm on $\mathrm{EHRs}^{33}$. Across all studies, accuracy was reported in about half of the instances and the ROC AUC was one of the most frequently reported outcomes.

Sensitivity and specificity were reported together in 10 studies. Blecker et al. $2016^{38}$ reported sensitivity together with PPV. Sensitivity and specificity were not measured in the study by Sideris et al. 2016 $6^{37}$, instead model accuracy and the ROC AUC were preferred. This study was concerned with developing an alternative 'comorbidity' framework based on disease and symptom diagnostic codes to cluster individuals at low to high risk of developing chronic heart failure.

PPVs were reported in six studies and accompanied with negative predictive values in two studies. These studies developed and validated machine-learning algorithms for the early detection of less investigated health conditions, these being hemodynamic instability in children ${ }^{19}$ and acute decompensated heart failure ${ }^{39}$. The highest number of outcome measures, including likelihood ratios, was observed in Calvert et al. $2016^{40}$ who investigated an under-represented population of patients with Alcohol Use Disorder.

The outcomes measured are summarized in Table 4.

Ongoing studies. Five studies are currently ongoing, one in Germany $^{43}$ and the others in the $\mathrm{USA}^{44-47}$. Two studies are prospective case series ${ }^{44,47}$, two studies are prospective cohort studies $^{43,45}$ and one is a RCT ${ }^{46}$. Two of the studies are concerned with developing prediction models, and the others are concerned with implementing machine learning algorithms into clinical practice as early warning systems.

The details of these trials are summarized in Table 5.

\section{Respiratory distress/failure}

The search yielded 1279 hits. Screening the titles and abstracts lead to 1142 being excluded. The full texts of the remaining 137 titles were obtained and assessed against the PICOS criteria. Studies were excluded due to irrelevant study design $(n=42)$, population $(n=6)$; intervention $(n=18)$ and outcomes $(n=47)$, and conference proceeding from before $2017(n=2)$. A total of 22 studies were finally included in this systematic literature review. None of the trials retrieved from ClinicalTrials.gov were included. The study selection process is depicted in Figure 2.

Study characteristics. Of the included studies, 17 were conducted in the US $\mathrm{US}^{33,48-63}$. Five studies were conducted outside the US; two in Canada ${ }^{64,65}$ by the same research group, two in France ${ }^{66,67}$ and one in the $\mathrm{UK}^{68}$. In total, 17 studies were retrospective ${ }^{33,48-50,52-55,58-66}$ and five were prospective $\mathrm{e}^{51,56,57,67,68}$. Of these studies, 12 were single-center $r^{33,48,49,51,52,54,55,58,59,64-66}$ and 10 studies were multi-center ${ }^{50,53,56,57,60-63,67,68}$. Five studies were time-series ${ }^{48,52,55,56,64}, 14$ studies were case-series ${ }^{33,49,51,53,54,57-62,65,66,68}$, one was case-control ${ }^{50}$ and one was case/time series study ${ }^{63}$.

The smallest sample of 100 patients came from two singlecenter retrospective studies ${ }^{48,66}$. Ten studies had sample sizes of 101-1000 $33,49-53,57,63,67,68$; seven studies had sample sizes of $1001-10,000^{54,55,59,60,62,64,65}$; and three had sample sizes larger than $10,000^{56,58,61}$. The largest study included more than 50,000 patients admitted to the ED of two centers over a 3-year period $^{61}$. Several published studies did not report their in-patient setting. When reported, some evaluated data from different wards $^{5,59,64,65,68}$, and some included patients admitted only to the $\mathrm{ED}^{53,54,61,63}$, the $\mathrm{ICU}^{48,60,67}$ and the surgical ward ${ }^{33,51,55}$.

The characteristics of all published studies are given in Table 6 .

CDS systems. About half of the studies developed machinelearning algorithms, whereas the other half focused on natural language processing (NLP) algorithms. One study differed from the rest by developing a computer-aided detection (CAD) system to measure the axial diameter of the right and left pulmonary ventricles, aiding in the diagnosis of pulmonary embolisms ${ }^{49}$. Many learning algorithms were concerned with detecting pulmonary embolisms and deep vein thrombosis ${ }^{53,54,58,59,64-67}$ as well as pneumonia ${ }^{33,48,57,60-63}$. Three studies developed machinelearning algorithms to detect $\mathrm{COPD}^{50,56,69}$. One study developed a machine learning algorithm to detect acute respiratory distress syndrome $^{52}$; while other studies developed machine learning algorithms to detect respiratory distress or failure following a pressure support ventilation trial $l^{67}$, cardiovascular surgery ${ }^{55}$ and pediatric tonsillectomy ${ }^{51}$. 
Table 2. Design aspects of published studies on shock.

\begin{tabular}{|c|c|c|c|c|c|c|}
\hline Study & Study Design & $\begin{array}{l}\text { Country and } \\
\text { institution(s) }\end{array}$ & $\begin{array}{l}\text { Number } \\
\text { of } \\
\text { patients } \\
\text { (records) }\end{array}$ & $\begin{array}{l}\text { Population/disease } \\
\text { definition }\end{array}$ & $\begin{array}{l}\text { In- } \\
\text { patient } \\
\text { setting }\end{array}$ & Collected data \\
\hline Ghosh 2017 & $\begin{array}{l}\text { Retrospective time } \\
\text { series } \\
\text { single center }\end{array}$ & $\begin{array}{l}\text { Australia } \\
\text { University of } \\
\text { Technology Sydney } \\
\text { \& The University of } \\
\text { Melbourne }\end{array}$ & 209 & $\begin{array}{l}\text { Sepsis or severe } \\
\text { sepsis }\end{array}$ & $\mathrm{ICU}$ & $\begin{array}{l}\text { (mean arterial pressure), } \\
\text { heart rate, respiratory } \\
\text { rate }\end{array}$ \\
\hline Hu 2016 & $\begin{array}{l}\text { Retrospective case } \\
\text { series } \\
\text { single center }\end{array}$ & $\begin{array}{l}\text { USA, Minnesota } \\
\text { University of Minnesota }\end{array}$ & NR (8909) & NR & Surgery & EHRs \\
\hline Li 2014 & $\begin{array}{l}\text { Retrospective case } \\
\text { series } \\
\text { multi-centric ( } 3 \\
\text { centers) }\end{array}$ & $\begin{array}{l}\text { UK, Oxford } \\
\text { University of Oxford \& } \\
\text { Mindray }\end{array}$ & NR (67) & $\begin{array}{l}\text { Ventricular flutter, } \\
\text { fibrillation and } \\
\text { tachycardia }\end{array}$ & NR & Electrocardiography \\
\hline Mahajan 2014 & $\begin{array}{l}\text { Prospective case } \\
\text { series } \\
\text { multi-centric ( } 4 \\
\text { centers) }\end{array}$ & $\begin{array}{l}\text { USA } \\
\text { University of Southern } \\
\text { California, Mayo Clinic- } \\
\text { Rochester, University of } \\
\text { North Carolina, Sanger } \\
\text { Heart \& Vascular } \\
\text { Institute \& Boston } \\
\text { Scientific }\end{array}$ & $410(908)$ & $\begin{array}{l}\text { Ventricular } \\
\text { fibrillation, ventricular } \\
\text { tachycardia and other } \\
\text { arrhythmias }\end{array}$ & NR & Electrograms \\
\hline Mao 2018 & $\begin{array}{l}\text { Retrospective case } \\
\text { series } \\
\text { multi-centric ( } 5 \\
\text { centers) }\end{array}$ & $\begin{array}{l}\text { USA } \\
\text { University of California, } \\
\text { Stanford Medical } \\
\text { Centre, Oroville } \\
\text { Hospital, Bakersfield } \\
\text { Heart Hospital, Cape } \\
\text { Regional Medical } \\
\text { Centre, Beth Israel } \\
\text { Deaconess Medical } \\
\text { Center }\end{array}$ & 359,390 & NR & various & Vital signs \\
\hline Reljin 2018 & $\begin{array}{l}\text { Prospective case- } \\
\text { control } \\
\text { multi-centric ( } 2 \\
\text { centers) }\end{array}$ & $\begin{array}{l}\text { USA } \\
\text { University of } \\
\text { Connecticut, Campbell } \\
\text { University School of } \\
\text { Medicine, University } \\
\text { of Massachusetts } \\
\text { Medical School,Yale } \\
\text { University School of } \\
\text { Medicine \& Worcester } \\
\text { Polytechnic Institute }\end{array}$ & $36(94)$ & $\begin{array}{l}\text { Traumatic injury, } \\
\text { healthy controls }\end{array}$ & NR & $\begin{array}{l}\text { Photoplethysmographic } \\
\text { signals }\end{array}$ \\
\hline Sideris 2016 & $\begin{array}{l}\text { Retrospective case } \\
\text { series } \\
\text { single center }\end{array}$ & $\begin{array}{l}\text { USA, Los Angeles } \\
\text { University of California }\end{array}$ & 1948 & Primarily heart failure & various & EHRs \\
\hline Blecker 2016 & $\begin{array}{l}\text { Retrospective case } \\
\text { series } \\
\text { single center }\end{array}$ & $\begin{array}{l}\text { USA, New York } \\
\text { NewYork-Presbyterian } \\
\text { Hospital \& New York } \\
\text { University }\end{array}$ & $\begin{array}{l}\text { NR } \\
(47,119)\end{array}$ & NR & various & EHRs \\
\hline Blecker 2018 & $\begin{array}{l}\text { Retrospective case } \\
\text { series } \\
\text { single center }\end{array}$ & $\begin{array}{l}\text { USA, New York } \\
\text { New York University }\end{array}$ & $\begin{array}{l}\text { NR } \\
(37229)\end{array}$ & NR & various & EHRs \\
\hline
\end{tabular}




\begin{tabular}{|c|c|c|c|c|c|c|}
\hline Study & Study Design & $\begin{array}{l}\text { Country and } \\
\text { institution(s) }\end{array}$ & $\begin{array}{l}\text { Number } \\
\text { of } \\
\text { patients } \\
\text { (records) }\end{array}$ & $\begin{array}{l}\text { Population/disease } \\
\text { definition }\end{array}$ & $\begin{array}{l}\text { In- } \\
\text { patient } \\
\text { setting }\end{array}$ & Collected data \\
\hline Calvert 2016 & $\begin{array}{l}\text { Retrospective time } \\
\text { series } \\
\text { single center }\end{array}$ & $\begin{array}{l}\text { USA, California } \\
\text { Dascena Inc. \& } \\
\text { University of California }\end{array}$ & 29083 & NR & $\mathrm{ICU}$ & vital signs \\
\hline Donald 2018 & $\begin{array}{l}\text { Retrospective } \\
\text { time series + } \\
\text { Prospective time } \\
\text { series } \\
\text { multi-centric ( } 22 \\
\text { centers) }\end{array}$ & Europe & 173 & Traumatic brain injury & $\mathrm{ICU}$ & $\begin{array}{l}\text { Demographic, clinical } \\
\text { and physiological data }\end{array}$ \\
\hline $\begin{array}{l}\text { Ebrahimzadeh } \\
2018\end{array}$ & $\begin{array}{l}\text { Retrospective time } \\
\text { series } \\
\text { single center }\end{array}$ & $\begin{array}{l}\text { Iran } \\
\text { University of Tehran, } \\
\text { Iran University } \\
\text { of Science and } \\
\text { Technology, University } \\
\text { of Sheikhbahaee } \\
\text { \& Payame Noor } \\
\text { University of North } \\
\text { Tehran }\end{array}$ & $53(106)$ & $\begin{array}{l}\text { Paroxysmal atrial } \\
\text { fibrillation }\end{array}$ & NR & Electrocardiography \\
\hline Potes 2017 & $\begin{array}{l}\text { Retrospective case } \\
\text { series } \\
\text { multi-centric ( } 2 \\
\text { centers) }\end{array}$ & $\begin{array}{l}\text { USA, California \& UK, } \\
\text { London } \\
\text { Children`s Hospital Los } \\
\text { Angeles, St. Mary`s } \\
\text { Hospital, London \& } \\
\text { Philips }\end{array}$ & 8022 & NR & ICU & $\begin{array}{l}\text { Vital signs, laboratory } \\
\text { values, and ventilator } \\
\text { parameters. }\end{array}$ \\
\hline Henry 2015 & $\begin{array}{l}\text { Retrospective case } \\
\text { series } \\
\text { single center }\end{array}$ & $\begin{array}{l}\text { USA, Maryland } \\
\text { John Hopkins } \\
\text { University }\end{array}$ & 16234 & NR & $\mathrm{ICU}$ & EHRs \\
\hline $\begin{array}{l}\text { Strodthoff } \\
2018\end{array}$ & $\begin{array}{l}\text { Retrospective time } \\
\text { series } \\
\text { single center }\end{array}$ & $\begin{array}{l}\text { Germany, Berlin } \\
\text { Fraunhofer Heinrich } \\
\text { Hertz Institute \& } \\
\text { University Medical } \\
\text { Center Schleswig- } \\
\text { Holstein, Kiel }\end{array}$ & $200(228)$ & $\begin{array}{l}\text { Myocardial infarction } \\
\text { and healthy controls }\end{array}$ & NR & Electrocardiography \\
\hline
\end{tabular}

USA: United States of America. UK: United Kingdom. NR: Not reported. ICU: Intensive care unit. EHR: Electronic health records.

The classifiers used in the NLP-based studies were various. However, some commonalities emerged between the studies developing machine-learning algorithms. Multiple studies applied SVM, logistic regression, random forests, K- nearest neighbor $(\mathrm{kNN})$, gradient boosting and neural network models. Various classifiers were explored in 5 studies. An overview of the developed learning algorithms is provided in Table 7.

One study, Reamoroon et al. $2018^{52}$, used a novel sampling technique to accommodate for inter-dependency in longitudinal data. Model accuracy and ROC AUC with this method was $<5 \%$ better than random sampling and $4-11 \%$ better than no sampling.

Outcome measures. The majority of the studies reported multiple outcome measures of model performance. The most frequently reported outcome measure was sensitivity, followed by specificity and ROC AUC. Likelihood ratios, on the other hand, were only reported in one study: Silva et al. $2017^{67}$ reported eight outcome measures of their novel machine learning model to predict post extubation distress. The outcomes measured across all studies are summarized in Table 8 .

Many of the studies that developed NLP-based algorithms reported negative and positive predictive values, as well as sensitivity and specificity. In contrast, the ROC AUC was the most frequently reported outcome measure of machine learning algorithm performance. It was also the single preferred outcome in three studies ${ }^{33,50,55}$. About half of the studies additionally reported sensitivity, specificity, and accuracy. One study reported specificity with sensitivity set at $90 \%$ and $95 \%$ to ensure that few disease positive cases were missed ${ }^{52}$. The single study that developed a CAD system measured the ROC AUC and model accuracy $^{49}$. 
Table 3. Overview of the algorithms developed to detect shock.

\begin{tabular}{|c|c|c|c|c|c|c|c|c|c|c|c|c|c|c|}
\hline \multirow[b]{2}{*}{ Study } & \multirow[b]{2}{*}{ Predicted disease } & \multicolumn{13}{|c|}{ Learning algorithm } \\
\hline & & 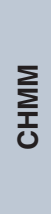 & 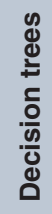 & 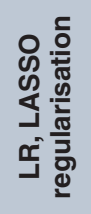 & 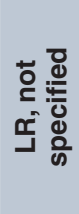 & $\sum_{\infty}$ & $\sum_{\underline{x}}$ & $\stackrel{\vec{u}}{\check{x}}$ & 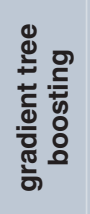 & 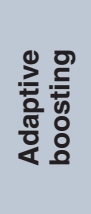 & 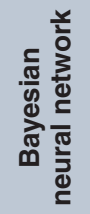 & 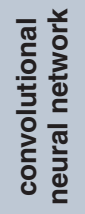 & 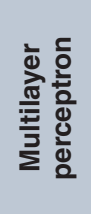 & 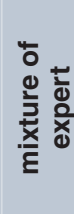 \\
\hline $\begin{array}{l}\text { Ebrahimzadeh } \\
2018\end{array}$ & $\begin{array}{l}\text { paroxysmal atrial } \\
\text { fibrillation }\end{array}$ & & & & & $\checkmark$ & $\checkmark$ & & & & & & $\checkmark$ & $\checkmark$ \\
\hline Li 2014 & $\begin{array}{l}\text { Ventricular fibrillation } \\
\text { and tachycardia }\end{array}$ & & & & & $\checkmark$ & & & & & & & & \\
\hline Mahajan 2014 & heart arrhythmias & & & & & $\checkmark$ & & & & & & & & \\
\hline $\begin{array}{l}\text { Strodthoff } \\
2018\end{array}$ & $\begin{array}{l}\text { myocardial } \\
\text { infarction }\end{array}$ & & & & & & & & & & & $\checkmark$ & & \\
\hline Sideris 2016 & heart failure & & & & & $\checkmark$ & & & & & & & & \\
\hline Blecker 2016 & heart failure & & & $\checkmark$ & & & & & & & & & & \\
\hline Blecker 2018 & heart failure & & & $\checkmark$ & & & & & & & & & & \\
\hline Reljin 2018 & Hypovolemia & & & & & $\checkmark$ & & & & & & & & \\
\hline Potes 2017 & $\begin{array}{l}\text { hemodynamic } \\
\text { instability }\end{array}$ & & & & & & & & & $\checkmark$ & & & & \\
\hline Donald 2018 & Hypotension & & & & & & & & & & $\checkmark$ & & & \\
\hline Ghosh 2017 & septic shock & $\checkmark$ & & & & & & & & & & & & \\
\hline Hu 2016 & septic shock & & & $\checkmark$ & & & & & & & & & & \\
\hline Mao 2018 & septic shock & & & & & & & & $\checkmark$ & & & & & \\
\hline Calvert 2016 & septic shock & & & & $\checkmark$ & & & & & & & & & \\
\hline Henry 2015 & septic shock & & & $\checkmark$ & & & & & & & & & & \\
\hline
\end{tabular}

CHMM: clustered hidden Markov model. LR: Logistic regression. SVM: Support vector machine. kNN: k nearest neighbor. RF: Random forest. Conv.: Convolutional.

Table 4. Overview of measured outcomes in studies on shock.

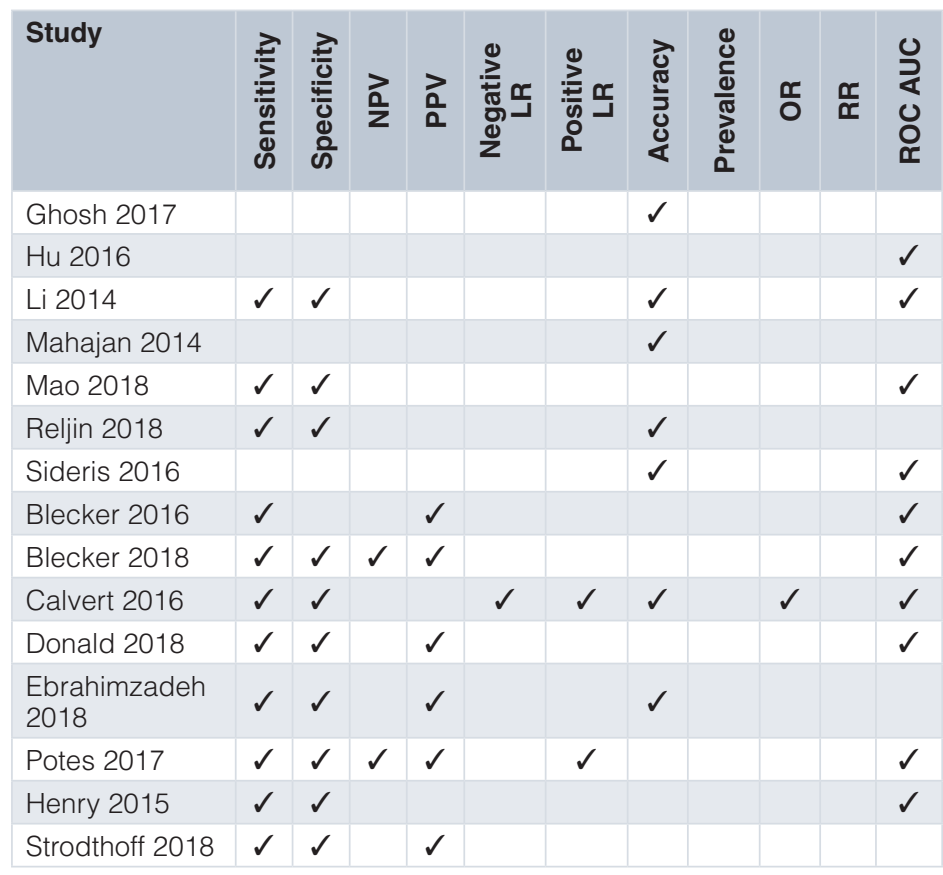

NPV: Negative predictive value. PPV: Positive predictive value. LR: Likelihood ratio. OR: Odds ratio. RR: Risk ratio. ROC AUC: Receiver operating characteristic area under the curve. 
Table 5. Overview of ongoing studies on shock.

\begin{tabular}{|c|c|c|c|c|c|c|}
\hline Identifier code & Study Design & $\begin{array}{l}\text { Countries } \\
\text { and study } \\
\text { centers }\end{array}$ & $\begin{array}{l}\text { Hospital } \\
\text { setting }\end{array}$ & Intervention & $\begin{array}{l}\text { Sample } \\
\text { characteristics }\end{array}$ & Outcome(s) \\
\hline NCT03582501 & $\begin{array}{l}\text { Prospective } \\
\text { case series } \\
\text { Year of study: } \\
\text { 2019-20 } \\
\text { Duration: } 12 \\
\text { months }\end{array}$ & $\begin{array}{l}\text { USA } \\
\text { Mayo Clinic } \\
\text { Arizona, } \\
\text { Florida \& } \\
\text { Rochester }\end{array}$ & NR & $\begin{array}{l}\text { Lower body } \\
\text { negative pressure } \\
\text { to simulate } \\
\text { hypovolemia }\end{array}$ & $\begin{array}{l}\text { Estimated: } 24 \\
\text { Age: 18-55 } \\
\text { Definition: Healthy } \\
\text { non-smoker, } \\
\text { no history of } \\
\text { hypertension, } \\
\text { diabetes, CAD } \\
\text { and neurologic } \\
\text { diseases }\end{array}$ & $\begin{array}{l}\frac{\text { Primary outcome }}{\text { Blood pressure }} \\
\text { Secondary outcome } \\
\text { Heart rate }\end{array}$ \\
\hline NCT02934971 & $\begin{array}{l}\text { Prospective } \\
\text { cohort study } \\
\text { Year of study: } \\
2017-19 \\
\text { Duration: } 24 \\
\text { months (up } \\
\text { to } 6 \text { months } \\
\text { follow-up) }\end{array}$ & $\begin{array}{l}\text { Germany, } \\
\text { Aachen } \\
\text { Aachen } \\
\text { University } \\
\text { Hospital }\end{array}$ & Out-patient & $\begin{array}{l}\text { Chemotherapy or } \\
\text { no chemotherapy }\end{array}$ & $\begin{array}{l}\text { Estimated: } 400 \\
\text { Age: } \geq 18 \\
\text { Definition: Patients } \\
\text { scheduled for } \\
\text { chemotherapy } \\
\text { at increased risk } \\
\text { of cardiotoxicity } \\
\text { and age-matched } \\
\text { controls }\end{array}$ & $\begin{array}{l}\text { Primary outcome } \\
\text { change in left ventricular } \\
\text { ejection fraction }\end{array}$ \\
\hline NCT03235193 & $\begin{array}{l}\text { Prospective } \\
\text { cohort study } \\
\text { Year of study: } \\
2017 \\
\text { Duration: } 3 \\
\text { months }\end{array}$ & $\begin{array}{l}\text { USA, West } \\
\text { Virginia } \\
\text { Dascena } \\
\text { Inc.\& } \\
\text { University of } \\
\text { California }\end{array}$ & $\mathrm{ED}, \mathrm{ICU}$ & $\begin{array}{l}\text { The InSight } \\
\text { algorithm used } \\
\text { as an EWS to } \\
\text { detect sepsis and } \\
\text { severe sepsis } \\
\text { detection from } \\
\text { EHRs compared } \\
\text { to severe sepsis } \\
\text { detection from } \\
\text { EHRs alone }\end{array}$ & $\begin{array}{l}\text { Estimated: } 1241 \\
\text { Age: } \geq 18 \\
\text { Definition: All } \\
\text { admitted patients }\end{array}$ & $\begin{array}{l}\text { Primary outcome } \\
\text { in-hospital mortality } \\
\text { Secondary outcomes } \\
\text { length of stay in hospital } \\
\text { and ICU, hospital } \\
\text { readmission }\end{array}$ \\
\hline NCT03644940 & $\begin{array}{l}\text { RCT } \\
\text { Year of study: } \\
\text { 2020-21 } \\
\text { Duration: } \\
6 \text { months }\end{array}$ & $\begin{array}{l}\text { USA, } \\
\text { California } \\
\text { Dascena } \\
\text { Inc.\& } \\
\text { University of } \\
\text { California }\end{array}$ & $\begin{array}{l}\text { Cardiology, } \\
\text { GI, ICU, } \\
\text { Medicine, } \\
\text { Oncology, } \\
\text { Surgery, } \\
\text { Transplant } \\
\text { and ED }\end{array}$ & $\begin{array}{l}\text { subpopulation- } \\
\text { optimized } \\
\text { version of InSight } \\
\text { compared to the } \\
\text { original version } \\
\text { used as an early } \\
\text { warning system to } \\
\text { identify patients at } \\
\text { high risk of severe } \\
\text { sepsis; followed } \\
\text { by physician } \\
\text { assessment of } \\
\text { sepsis }\end{array}$ & $\begin{array}{l}\text { Estimated n: } 51645 \\
\text { Age: }>18 \\
\text { Definition: NR }\end{array}$ & $\begin{array}{l}\text { Primary outcomes } \\
\text { in-hospital SIRS-based } \\
\text { mortality } \\
\text { Secondary outcomes } \\
\text { in-hospital severe sepsis/ } \\
\text { shock-coded mortality; } \\
\text { SIRS-based hospital } \\
\text { length of stay; Severe } \\
\text { sepsis/shock-coded } \\
\text { hospital length of stay }\end{array}$ \\
\hline NCT03655626 & $\begin{array}{l}\text { Single-arm } \\
\text { trial up to } \\
\text { Year of study: } \\
\text { 2018-19 up to } \\
\text { Duration: } \\
6 \text { months }\end{array}$ & $\begin{array}{l}\text { USA, North } \\
\text { Carolina } \\
\text { Duke } \\
\text { University } \\
\text { Hospital }\end{array}$ & ED & $\begin{array}{l}\text { machine learning } \\
\text { algorithm to } \\
\text { predict sepsis, } \\
\text { custom dashboard } \\
\text { and monitoring }\end{array}$ & $\begin{array}{l}\text { Estimated n: } 3200 \\
\text { Age: }>18 \\
\text { Definition: NR }\end{array}$ & $\begin{array}{l}\text { Primary outcome } \\
\text { rate of CMS bundle } \\
\text { completion for patients } \\
\text { with sepsis } \\
\text { Secondary outcomes } \\
\text { time to sepsis diagnosis; } \\
\text { number of patients } \\
\text { developing sepsis; } \\
\text { number of patients } \\
\text { developing sepsis and } \\
\text { not treated; length of } \\
\text { stay in ED and hospital; } \\
\text { inpatient mortality; ICU } \\
\text { requirement rate; time } \\
\text { from sepsis onset to } \\
\text { blood culture, antibiotics, } \\
\text { IV fluids, lactate, CMS } \\
\text { bundle completion; rate } \\
\text { of lactate complete; } \\
\text { number of sepsis } \\
\text { diagnostic codes per } \\
\text { month }\end{array}$ \\
\hline
\end{tabular}




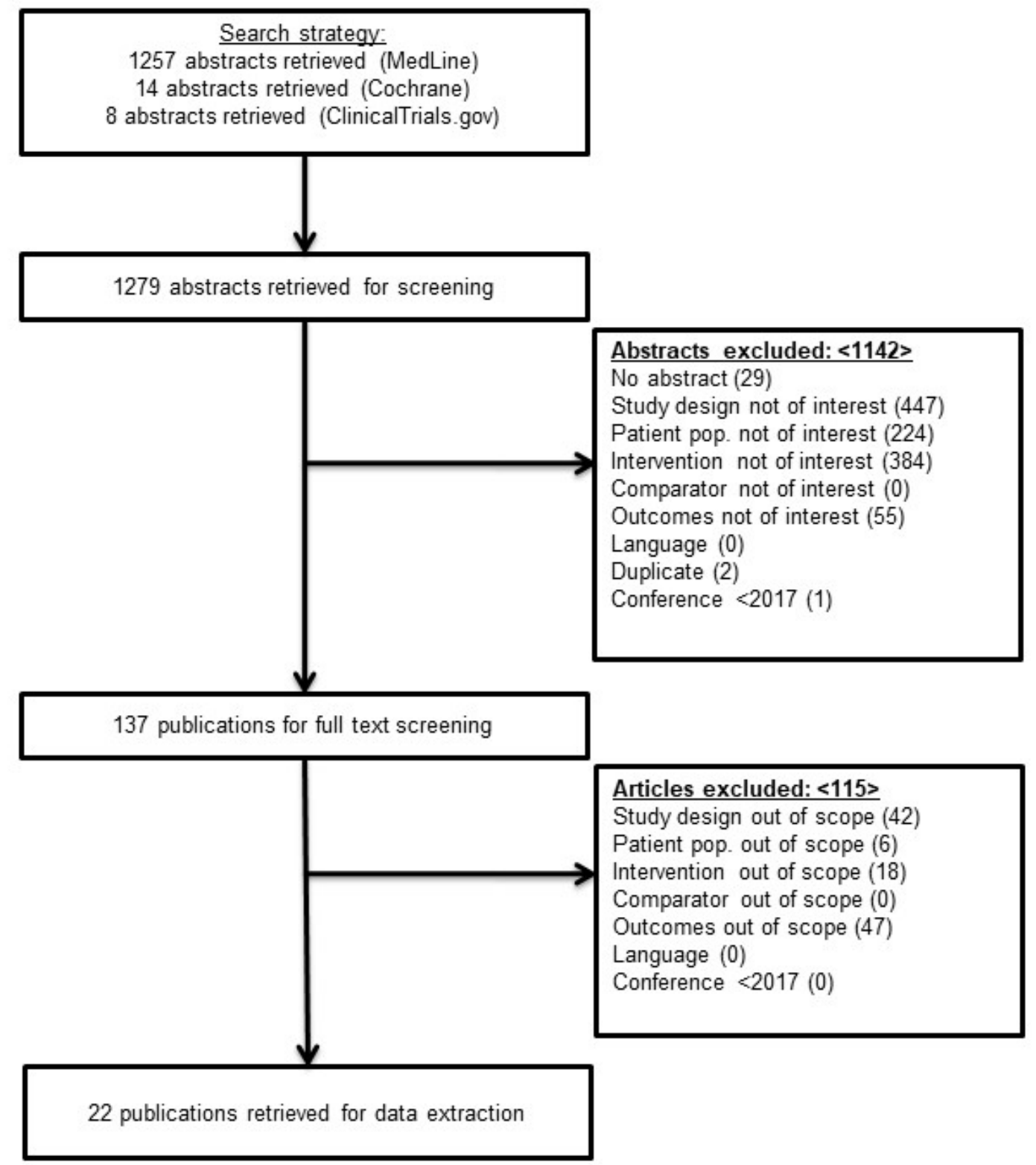

Figure 2. Study selection - Respiratory distress-failure. Pop. = Population. 
Table 6. Design aspects of published studies on respiratory distress or failure.

\begin{tabular}{|c|c|c|c|c|c|}
\hline Study & Study Design & Countries and institution(s) & $\begin{array}{l}\text { Number of } \\
\text { patients } \\
\text { (records) }\end{array}$ & Population/disease definition & $\begin{array}{l}\text { In-patient } \\
\text { setting }\end{array}$ \\
\hline Bejan 2013 & $\begin{array}{l}\text { Retrospective time } \\
\text { series } \\
\text { single center }\end{array}$ & $\begin{array}{l}\text { USA, Washington } \\
\text { University of Washington }\end{array}$ & 100 & NR & $\mathrm{ICU}$ \\
\hline $\begin{array}{l}\text { Kumamaru } \\
2016\end{array}$ & $\begin{array}{l}\text { Retrospective case } \\
\text { series } \\
\text { single center }\end{array}$ & $\begin{array}{l}\text { USA, Massachusetts } \\
\text { Brigham and Women's Hospital }\end{array}$ & 125 & acute pulmonary embolism & NR \\
\hline $\begin{array}{l}\text { Bodduluri } \\
2013\end{array}$ & $\begin{array}{l}\text { Retrospective } \\
\text { case-control } \\
\text { multi-center } \\
\text { (national data) }\end{array}$ & $\begin{array}{l}\text { USA, lowa } \\
\text { The University of lowa }\end{array}$ & 153 & $\begin{array}{l}\text { smokers with or without COPD } \\
\text { and non-smokers }\end{array}$ & NR \\
\hline Biesiada 2014 & $\begin{array}{l}\text { Prospective case } \\
\text { series } \\
\text { single center }\end{array}$ & $\begin{array}{l}\text { USA, Cincinnati } \\
\text { Children's Hospital Medical } \\
\text { Center \& University of Cincinnati }\end{array}$ & 347 & $\begin{array}{l}\text { current tonsillitis, adenotonsillar } \\
\text { hypertrophy or obstructive sleep } \\
\text { apnea }\end{array}$ & Surgery \\
\hline $\begin{array}{l}\text { Reamaroon } \\
2018\end{array}$ & $\begin{array}{l}\text { Retrospective time } \\
\text { series } \\
\text { single-center }\end{array}$ & $\begin{array}{l}\text { USA, Michigan } \\
\text { University of Michigan }\end{array}$ & 401 & $\begin{array}{l}\text { mild hypoxia and acute hypoxic } \\
\text { respiratory failure }\end{array}$ & NR \\
\hline Vinson 2015 & $\begin{array}{l}\text { Retrospective case } \\
\text { series } \\
\text { multi-center ( } 4 \\
\text { centers) }\end{array}$ & $\begin{array}{l}\text { USA, California } \\
\text { the Kaisers Permanente CREST } \\
\text { Network }\end{array}$ & 593 & acute pulmonary embolism & ED \\
\hline Huesch 2018 & $\begin{array}{l}\text { Retrospective case } \\
\text { series } \\
\text { single center }\end{array}$ & $\begin{array}{l}\text { USA, Pennsylvania } \\
\text { Milton S. Hershey Medical Center }\end{array}$ & 1133 & $\begin{array}{l}\text { individuals suspected of } \\
\text { pulmonary embolism }\end{array}$ & ED \\
\hline $\begin{array}{l}\text { Mortazavi } \\
2017\end{array}$ & $\begin{array}{l}\text { Retrospective time } \\
\text { series } \\
\text { single center }\end{array}$ & $\begin{array}{l}\text { USA, Connecticut } \\
\text { Yale University }\end{array}$ & 5214 & $\begin{array}{l}\text { patients undergoing } \\
\text { cardiovascular procedures: } \\
\text { CABG, } \mathrm{PCI} \text { and ICD procedures }\end{array}$ & Surgery \\
\hline Pham 2014 & $\begin{array}{l}\text { Retrospective case } \\
\text { series } \\
\text { single center }\end{array}$ & $\begin{array}{l}\text { France } \\
\text { CHU de Caen, Caen \& Hôpital } \\
\text { Européen Georges-Pompidou, } \\
\text { Paris }\end{array}$ & $N R(100)$ & $\begin{array}{l}\text { individuals suspected of having } \\
\text { Venous thromboembolism }\end{array}$ & NR \\
\hline $\begin{array}{l}\text { Rochefort } \\
2015\end{array}$ & $\begin{array}{l}\text { Retrospective time } \\
\text { series } \\
\text { single center }\end{array}$ & $\begin{array}{l}\text { Canada, Quebec } \\
\text { McGill University }\end{array}$ & $\begin{array}{l}1649 \\
(2000)\end{array}$ & $\begin{array}{l}\text { individuals suspected of having } \\
\text { Venous thromboembolism }\end{array}$ & various \\
\hline Silva 2017 & $\begin{array}{l}\text { Prospective } \\
\text { before-after } \\
\text { multi-center ( } 3 \\
\text { centers) }\end{array}$ & $\begin{array}{l}\text { France } \\
\text { University Teaching Hospital } \\
\text { of Purpan, Toulouse; Hopital Dieu } \\
\text { Hospital, Narbonne; Saint Eloi } \\
\text { Hospital, Montpellier }\end{array}$ & 136 & $\begin{array}{l}\text { hemodynamic instability, } \\
\text { respiratory failure, multiple } \\
\text { trauma, nontraumatic coma, and } \\
\text { postoperative complication of } \\
\text { abdominal surgery }\end{array}$ & $\mathrm{ICU}$ \\
\hline $\begin{array}{l}\text { Gonzalez } \\
2018\end{array}$ & $\begin{array}{l}\text { Prospective time } \\
\text { series } \\
\text { multi-center, multi- } \\
\text { national }\end{array}$ & $\begin{array}{l}\text { USA } \\
\text { Binham and Women`s Hospital } \\
\text { (on behalf of the COPD and } \\
\text { ECLIPSE Study investigators) }\end{array}$ & 11655 & smokers with or without COPD & various \\
\hline Tian 2017 & $\begin{array}{l}\text { Retrospective case } \\
\text { series } \\
\text { single center }\end{array}$ & $\begin{array}{l}\text { Canada, Quebec } \\
\text { Mcgill University }\end{array}$ & $\begin{array}{l}2819 \\
(4000)\end{array}$ & $\begin{array}{l}\text { individuals suspected of having } \\
\text { Venous thromboembolism }\end{array}$ & various \\
\hline
\end{tabular}




\begin{tabular}{|c|c|c|c|c|c|}
\hline Study & Study Design & Countries and institution(s) & $\begin{array}{l}\text { Number of } \\
\text { patients } \\
\text { (records) }\end{array}$ & Population/disease definition & $\begin{array}{l}\text { In-patient } \\
\text { setting }\end{array}$ \\
\hline Choi 2018 & $\begin{array}{l}\text { Prospective case } \\
\text { series } \\
\text { multi-center ( } 3 \\
\text { centers) }\end{array}$ & $\begin{array}{l}\text { USA } \\
\text { Mayo Clinic, Scottsdale; National } \\
\text { Jewish Health, Denve; University } \\
\text { of Washington Medical Center, } \\
\text { Seattle \& Veracyte Inc. }\end{array}$ & 139 (403) & suspected interstitial lung disease & NR \\
\hline Yu 2014 & $\begin{array}{l}\text { Retrospective case } \\
\text { series } \\
\text { single center }\end{array}$ & $\begin{array}{l}\text { USA, Massachusetts } \\
\text { Brigham, and Women's Hospital \& } \\
\text { Harvard Medical School, }\end{array}$ & $\begin{array}{l}\text { NR } \\
(10,330)\end{array}$ & $\begin{array}{l}\text { individuals suspected of } \\
\text { pulmonary embolism }\end{array}$ & NR \\
\hline Swartz 2017 & $\begin{array}{l}\text { Retrospective case } \\
\text { series } \\
\text { single center }\end{array}$ & $\begin{array}{l}\text { USA, New York } \\
\text { New York University \& Mount Sinai } \\
\text { St. Luke`s Hospital }\end{array}$ & NR (2400) & $\begin{array}{l}\text { individuals suspected of having } \\
\text { Venous thromboembolism }\end{array}$ & various \\
\hline Liu 2013 & $\begin{array}{l}\text { Retrospective case } \\
\text { series } \\
\text { multi-center }(21 \\
\text { centers) }\end{array}$ & $\begin{array}{l}\text { USA, California } \\
\text { Kaiser Permanente }\end{array}$ & NR (2466) & NR & $\mathrm{ICU}$ \\
\hline Haug 2013 & $\begin{array}{l}\text { Retrospective case } \\
\text { series } \\
\text { multi-center( } 2 \\
\text { centers) }\end{array}$ & $\begin{array}{l}\text { USA, Utah } \\
\text { LDS Hospital and Intermountain } \\
\text { Medical Centre }\end{array}$ & $\begin{array}{l}\text { NR } \\
(362,924)\end{array}$ & NR & ED \\
\hline Dublin 2013 & $\begin{array}{l}\text { Retrospective case } \\
\text { series } \\
\text { multi-center } \\
\text { (regional data) }\end{array}$ & $\begin{array}{l}\text { USA, Seattle } \\
\text { Group Health Research Institute \& } \\
\text { University of Washington }\end{array}$ & NR (5000) & NR & NR \\
\hline Phillips 2014 & $\begin{array}{l}\text { Prospective case } \\
\text { series } \\
\text { multi-center }\end{array}$ & $\begin{array}{l}\text { UK, Llaneli } \\
\text { Swansea University, Aberystwyth } \\
\text { University \& Hywel Dda University } \\
\text { Health Board }\end{array}$ & 181 & with and without COPD & various \\
\hline Hu 2016 & $\begin{array}{l}\text { Retrospective case } \\
\text { series } \\
\text { single center }\end{array}$ & $\begin{array}{l}\text { USA, Minnesota } \\
\text { University of Minnesota }\end{array}$ & NR (8909) & NR & Surgery \\
\hline Jones 2018 & $\begin{array}{l}\text { Retrospective } \\
\text { case/time series } \\
\text { multi-center } \\
\text { (number of centers } \\
\text { unknown) }\end{array}$ & $\begin{array}{l}\text { USA, Utah \& Washington } \\
\text { VA Salt Lake City Health Care } \\
\text { System, University of Utah \& } \\
\text { George Washington University }\end{array}$ & NR (911) & $\begin{array}{l}\text { individuals suspected of } \\
\text { pneumonia }\end{array}$ & ED \\
\hline
\end{tabular}

NA: Not applicable. NR: Not reported. USA: United States of America. COPD: Chronic obstructive pulmonary disease. ECLIPSE: Evaluations of COPD Longitudinally to Identify Predictive Surrogate Endpoints. UK: United Kingdom. CABG: Coronary artery bypass grafting. PCI: Percutaneous coronary intervention. ICD: Implantable cardioverter defibrillator. ICU: Intensive care unit. ED: Emergency department.

\section{Infection or sepsis}

The search yielded 2659 hits. Screening the titles and abstracts lead to 2562 being excluded. The full texts of the remaining 97 titles were obtained and assessed against the PICOS criteria. Studies were excluded due to irrelevant study design $(n=41)$, population $(n=4)$; intervention $(n=6)$ and outcomes $(n=14)$. A total of 31 studies were finally included in this systematic literature review. Four of these were ongoing trials. The study selection process is depicted in Figure 3.

Study characteristics. Of the included studies, 24 were conducted in the US. Three studies were conducted outside the US; one in France; one in the Netherlands and one in the UK. In total, 21 studies were retrospective $\mathrm{e}^{33,35,70-88}$ and six were prospective ${ }^{89-94}$. There were 21 single-center studies $^{33,70-75,77-83,86-88,90-92,94}$ and six multi-center studies ${ }^{35,76,84,85,89,93}$. Seven studies were time series $^{71,78,82,84-86,92}, 18$ studies were case series ${ }^{33,35,70,72-76,80,81,83,87-91,93,94}$, one was a case-control ${ }^{77}$ and one was a matched-controlled study ${ }^{79}$.

The smallest studies included patients with leukemia ${ }^{89}$ and combat casualty patients ${ }^{90}$. Four studies had a sample size below $1000^{70,72,73,79}$, three had a sample size between $1001-10,000^{33,71,87}$ and 12 had a sample size larger than $10,000^{35,74,77-78,80-82,84-87,88}$. 


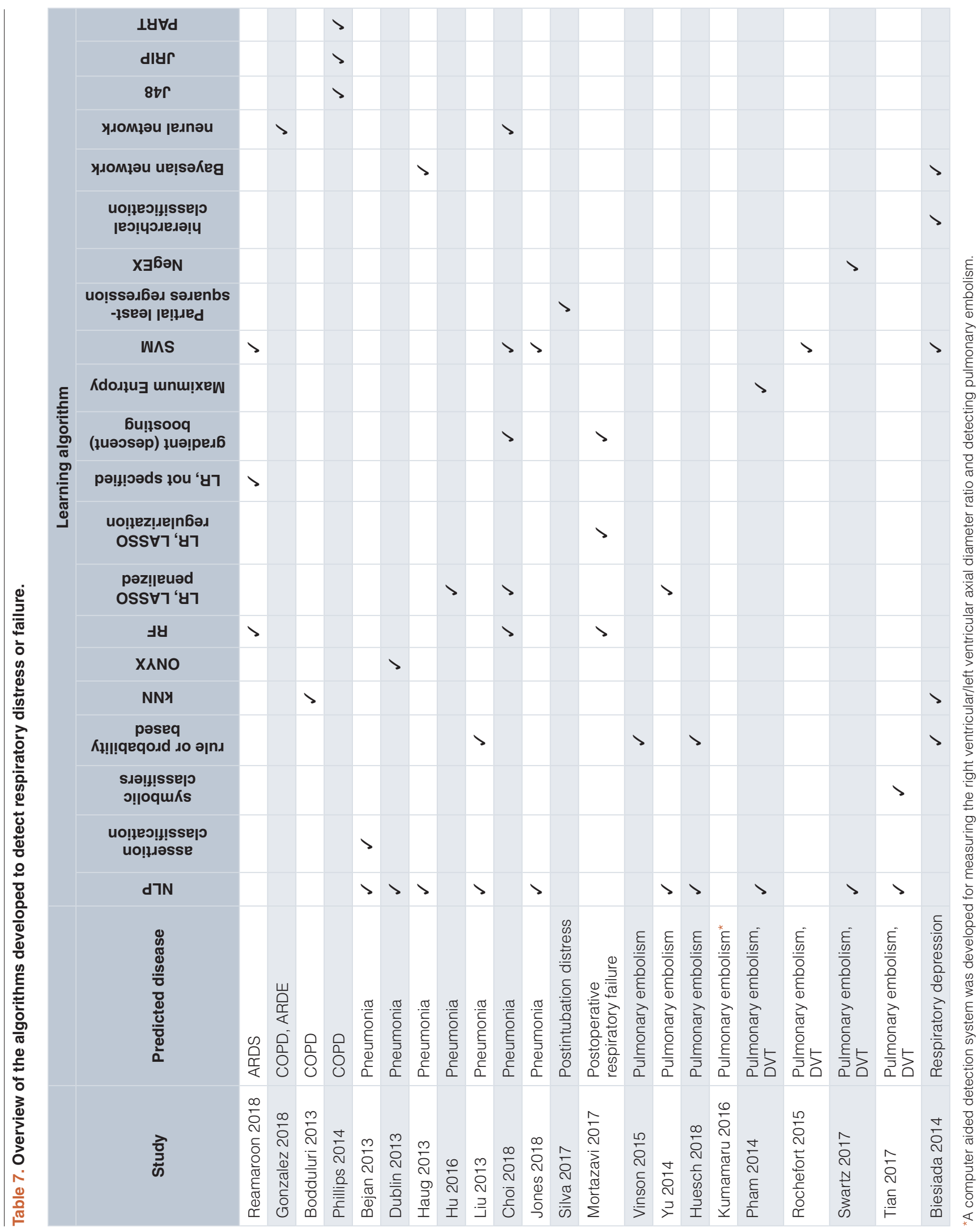


$\overline{\text { Table 8. Overview of measured outcomes in studies predicting respiratory distress or failure. }}$

\begin{tabular}{|c|c|c|c|c|c|c|c|c|c|c|c|c|c|}
\hline Study & Algorithm & & $\begin{array}{l}\frac{2}{0} \\
\frac{0}{0} \\
\text { के } \\
\text { के }\end{array}$ & $\frac{2}{z}$ & $\vec{a}$ & 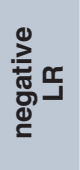 & $\sum_{\substack{0 \\
0}}^{0}$ & 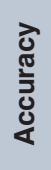 & 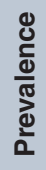 & ๕̃ & $\underset{\mathscr{\alpha}}{\widetilde{r}}$ & 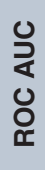 & $\begin{array}{l}\frac{0}{\bar{N}} \\
\frac{0}{0} \\
\frac{0}{0} \\
\frac{\pi}{0}\end{array}$ \\
\hline Kumamaru 2016 & CAD & & & & & & & $\checkmark$ & & & & $\checkmark$ & \\
\hline Bodduluri 2013 & ML & & & & & & & & & & & $\checkmark$ & \\
\hline Hu 2016 & ML & & & & & & & & & & & $\checkmark$ & \\
\hline Mortazavi 2017 & ML & & & & & & & & & & & $\checkmark$ & \\
\hline Rochefort 2015 & ML & $\checkmark$ & $\checkmark$ & $\checkmark$ & $\checkmark$ & & & & & & & $\checkmark$ & \\
\hline Silva 2017 & $M L$ & $\checkmark$ & $\checkmark$ & $\checkmark$ & $\checkmark$ & $\checkmark$ & $\checkmark$ & $\checkmark$ & & & & $\checkmark$ & \\
\hline Vinson 2015 & $M L$ & $\checkmark$ & $\checkmark$ & $\checkmark$ & $\checkmark$ & & & $\checkmark$ & & & & & \\
\hline Biesiada 2014 & $M L$ & $\checkmark$ & $\checkmark$ & & & & & $\checkmark$ & $\checkmark$ & & $\checkmark$ & & \\
\hline Choi 2018 & $M L$ & $\checkmark$ & $\checkmark$ & & & & & & & & & $\checkmark$ & \\
\hline Gonzalez 2018 & $M L$ & & & & & & & $\checkmark$ & $\checkmark$ & $\checkmark$ & & $\checkmark$ & \\
\hline Phillips 2014 & $M L$ & $\checkmark$ & $\checkmark$ & & & & & $\checkmark$ & & & & $\checkmark$ & \\
\hline Reamaroon 2018 & $M L$ & & $\checkmark$ & & & & & $\checkmark$ & & & & $\checkmark$ & \\
\hline Bejan 2013 & NLP & $\checkmark$ & $\checkmark$ & $\checkmark$ & $\checkmark$ & & & $\checkmark$ & & & & & \\
\hline Dublin 2013 & NLP & $\checkmark$ & $\checkmark$ & $\checkmark$ & $\checkmark$ & & & & & & & & \\
\hline Haug 2013 & NLP & & & & & & & & & & & $\checkmark$ & \\
\hline Liu 2013 & NLP & $\checkmark$ & $\checkmark$ & $\checkmark$ & $\checkmark$ & & & & & & & & \\
\hline Pham 2014 & NLP & $\checkmark$ & & & $\checkmark$ & & & & & & & & \\
\hline Swartz 2017 & NLP & $\checkmark$ & $\checkmark$ & $\checkmark$ & $\checkmark$ & & & & & & & & $\checkmark$ \\
\hline Tian 2017 & NLP & $\checkmark$ & $\checkmark$ & $\checkmark$ & $\checkmark$ & & & & & & & & \\
\hline Yu 2014 & NLP & & & $\checkmark$ & $\checkmark$ & & & & & & & $\checkmark$ & \\
\hline Huesch 2018 & NLP & $\checkmark$ & $\checkmark$ & $\checkmark$ & $\checkmark$ & & & $\checkmark$ & & & & & \\
\hline Jones 2018 & NLP & $\checkmark$ & $\checkmark$ & $\checkmark$ & $\checkmark$ & & & & & & & $\checkmark$ & \\
\hline
\end{tabular}

NLP: Natural language processing. ML: Machine learning. CAD: Computer aided detection. NPV: Negative predictive value. PPV: Positive predictive value. LR: Likelihood ratio. OR: Odds ratio. RR: Risk ratio. ROC AUC: Receiver operating characteristic area under the curve.

Eight studies had samples even larger than $50,000^{35,74,77,78,82,84,85,88}$. Large samples were achieved by less restrictive inclusion criteria where all patients admitted to specific ward(s) or hospital(s) over a given time were defined.

Majority of the published studies evaluated data from different wards; several studies included patients admitted only to the $\operatorname{ICU}^{70,72,81,84-86,93}$ and surgical ward ${ }^{73,76,78,87,91,92}$, less often the
General ward $^{33}$ and Emergency Department ${ }^{74}$. Of these, 23 studies included data collected at their own hospital; and four utilized previously collated databases ${ }^{76,81,84,86}$.

The characteristics of all published studies are given in Table 9.

CDS systems. The machine learning algorithms evaluated in the studies were developed to predict a range of diseases. These 


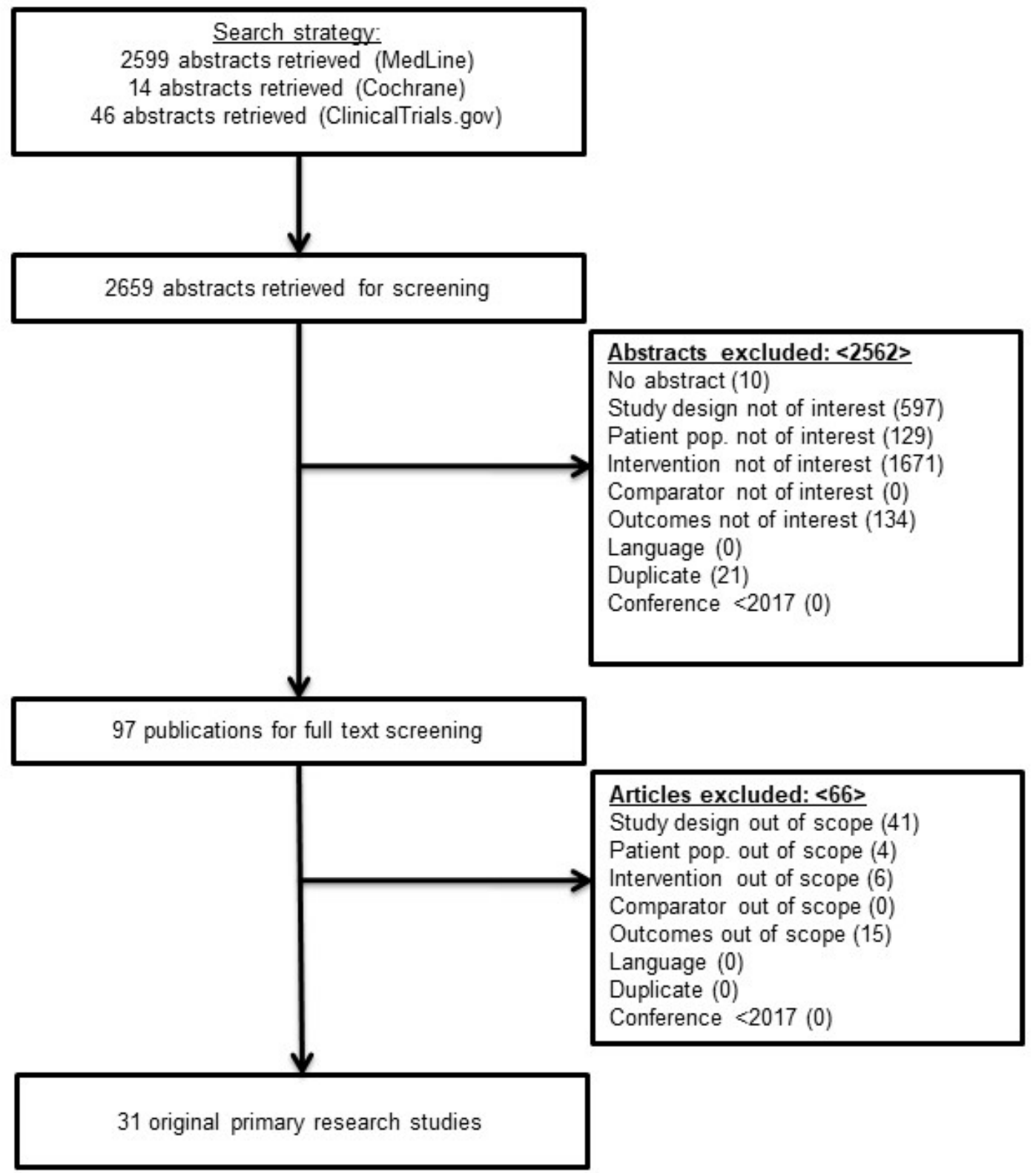

Figure 3. Study selection - infection or sepsis. Pop. = Population. 
Table 9. Design aspects of published studies on infection or sepsis.

\begin{tabular}{|c|c|c|c|c|c|}
\hline Study & Study Design & Country and institution(s) & $\begin{array}{l}\text { Number of } \\
\text { patients } \\
\text { (records) }\end{array}$ & $\begin{array}{l}\text { Population/disease } \\
\text { definition }\end{array}$ & $\begin{array}{l}\text { In-patient } \\
\text { setting }\end{array}$ \\
\hline Ahmed 2015 & $\begin{array}{l}\text { Retrospective case } \\
\text { series } \\
\text { single center }\end{array}$ & $\begin{array}{l}\text { USA, Minnesota } \\
\text { Mayo Clinic Rochester }\end{array}$ & 944 & NR & $\mathrm{ICU}$ \\
\hline Brasier, 2015 & $\begin{array}{l}\text { Prospective case } \\
\text { series } \\
\text { multi-center ( } 3 \\
\text { sites) }\end{array}$ & $\begin{array}{l}\text { USA, Texas } \\
\text { Aspergillus Technology } \\
\text { Consortium \& University of Texas }\end{array}$ & 57 & Leukemia & NR \\
\hline Dente, 2017 & $\begin{array}{l}\text { Prospective case } \\
\text { series } \\
\text { single center }\end{array}$ & $\begin{array}{l}\text { USA, Maryland } \\
\text { Emory University, Walter Reed } \\
\text { National Military Medical Centre }\end{array}$ & 73 & Combat casualty patients & NR \\
\hline $\mathrm{Hu}, 2016$ & $\begin{array}{l}\text { Retrospective case } \\
\text { series } \\
\text { single center }\end{array}$ & $\begin{array}{l}\text { USA, Minnesota } \\
\text { University of Minnesota }\end{array}$ & NR $(8,909)$ & NR & General \\
\hline Konerman, 2017 & $\begin{array}{l}\text { Retrospective time } \\
\text { series } \\
\text { single center }\end{array}$ & $\begin{array}{l}\text { USA, Michigan } \\
\text { University of Michigan }\end{array}$ & 1,233 & Chronic hepatitis c & NR \\
\hline Legrand, 2013 & $\begin{array}{l}\text { Prospective case } \\
\text { series } \\
\text { single center }\end{array}$ & $\begin{array}{l}\text { France, Paris } \\
\text { Hôpital Européen Georges } \\
\text { Pompidou Assistance Publique- } \\
\text { Hopitaux de Paris }\end{array}$ & 202 & Infective endocarditis & Surgery \\
\hline Mani, 2014 & $\begin{array}{l}\text { Retrospective case } \\
\text { series } \\
\text { single center }\end{array}$ & $\begin{array}{l}\text { USA, New Mexico } \\
\text { University of New Mexico }\end{array}$ & 299 & Sepsis & $\mathrm{ICU}$ \\
\hline Mao 2018 & $\begin{array}{l}\text { Retrospective case } \\
\text { series } \\
\text { multi-center ( } 5 \\
\text { centers) }\end{array}$ & $\begin{array}{l}\text { USA } \\
\text { University of California, Stanford } \\
\text { Medical Centre, Oroville Hospital, } \\
\text { Bakersfield Heart Hospital, Cape } \\
\text { Regional Medical Centre, Beth } \\
\text { Israel Deaconess Medical Center }\end{array}$ & 359,390 & NR & various \\
\hline Sanger, 2016 & $\begin{array}{l}\text { Prospective time } \\
\text { series } \\
\text { single center }\end{array}$ & $\begin{array}{l}\text { USA, Washington } \\
\text { University of Washington }\end{array}$ & 851 & $\begin{array}{l}\text { Open-abdominal surgery } \\
\text { patients }\end{array}$ & Surgery \\
\hline Scicluna, 2017 & $\begin{array}{l}\text { Prospective case } \\
\text { series } \\
\text { multi-center ( } 2 \\
\text { sites + national } \\
\text { database) }\end{array}$ & $\begin{array}{l}\text { Netherlands \& UK Amsterdam } \\
\text { Academic Medical Center, Utrecht } \\
\text { University Medical Center \& UK } \\
\text { Genomic Advances in Sepsis } \\
\text { study }\end{array}$ & 787 & Sepsis & $\mathrm{ICU}$ \\
\hline Sohn, 2016 & $\begin{array}{l}\text { Retrospective case } \\
\text { series } \\
\text { single center }\end{array}$ & $\begin{array}{l}\text { USA, Minnesota } \\
\text { Mayo Clinic Rochester }\end{array}$ & 751 & Colorectal surgery patients & Surgery \\
\hline Taylor, 2018 & $\begin{array}{l}\text { Retrospective case } \\
\text { series } \\
\text { single center }\end{array}$ & $\begin{array}{l}\text { USA, Connecticut } \\
\text { Yale University School of } \\
\text { Medicine, }\end{array}$ & $\begin{array}{l}55,365 \\
(80,387)\end{array}$ & $\begin{array}{l}\text { Suspected urine tract } \\
\text { infection }\end{array}$ & ED \\
\hline Hernandez 2017 & $\begin{array}{l}\text { Retrospective case } \\
\text { series } \\
\text { single center }\end{array}$ & $\begin{array}{l}\text { UK, London } \\
\text { Imperial College Healthcare NHS } \\
\text { Trust }\end{array}$ & $>500,000$ & NR & NR \\
\hline
\end{tabular}




\begin{tabular}{|c|c|c|c|c|c|}
\hline Study & Study Design & Country and institution(s) & $\begin{array}{l}\text { Number of } \\
\text { patients } \\
\text { (records) }\end{array}$ & $\begin{array}{l}\text { Population/disease } \\
\text { definition }\end{array}$ & $\begin{array}{l}\text { In-patient } \\
\text { setting }\end{array}$ \\
\hline $\begin{array}{l}\text { Bartz-Kurycki } \\
2018\end{array}$ & $\begin{array}{l}\text { Retrospective case } \\
\text { series } \\
\text { multi-center } \\
\text { (national database) }\end{array}$ & $\begin{array}{l}\text { USA, Texas } \\
\text { University of Texas }\end{array}$ & 13,589 & NR & Surgery \\
\hline Beeler 2018 & $\begin{array}{l}\text { Retrospective } \\
\text { case-control } \\
\text { single center }\end{array}$ & $\begin{array}{l}\text { USA, Indiana } \\
\text { Indiana University Health } \\
\text { Academic Health Center }\end{array}$ & $\operatorname{NR}(70,218)$ & $\begin{array}{l}\text { Central venous line with } \\
\text { or without central line- } \\
\text { associated bloodstream } \\
\text { infections }\end{array}$ & NR \\
\hline Bihorac 2018 & $\begin{array}{l}\text { Retrospective time } \\
\text { series } \\
\text { single center }\end{array}$ & $\begin{array}{l}\text { USA, Florida } \\
\text { University of Florida Health }\end{array}$ & 51,457 & NR & Surgery \\
\hline Chen 2018 & $\begin{array}{l}\text { Retrospective } \\
\text { matched pairs ( } 1: 1 \\
\text { case matching) } \\
\text { single center }\end{array}$ & $\begin{array}{l}\text { USA, Kansas } \\
\text { University of Kansas Health } \\
\text { System }\end{array}$ & 358 & $\begin{array}{l}\text { Stage } 3 \mathrm{AKI} \text { and non-AKI } \\
\text { controls }\end{array}$ & NR \\
\hline Cheng 2017 & $\begin{array}{l}\text { Retrospective case } \\
\text { series } \\
\text { single center }\end{array}$ & $\begin{array}{l}\text { USA, Kansas } \\
\text { University of Kansas Medical } \\
\text { Center }\end{array}$ & $\begin{array}{l}33,703 \\
(48,955)\end{array}$ & NR & NR \\
\hline Desautels 2016 & $\begin{array}{l}\text { Retrospective case } \\
\text { series } \\
\text { single center }\end{array}$ & $\begin{array}{l}\text { USA, California } \\
\text { Dascena Inc.\& University of } \\
\text { California }\end{array}$ & $\operatorname{NR}(21,176)$ & NR & $\mathrm{ICU}$ \\
\hline Koyner 2015 & $\begin{array}{l}\text { Retrospective time } \\
\text { series } \\
\text { single center }\end{array}$ & $\begin{array}{l}\text { USA, Chicago University of } \\
\text { Chicago }\end{array}$ & NR $(121,158)$ & NR & NR \\
\hline LaBarbera 2015 & $\begin{array}{l}\text { Retrospective case } \\
\text { series } \\
\text { single center }\end{array}$ & $\begin{array}{l}\text { USA, Pennsylvania } \\
\text { Pinnacle Health Hospital, } \\
\text { Harrisburg }\end{array}$ & 198 & Clostridium difficile infection & NR \\
\hline $\begin{array}{l}\text { Mohamadlou } \\
2018\end{array}$ & $\begin{array}{l}\text { Retrospective time } \\
\text { series } \\
\text { multi-center ( } 2 \\
\text { sites) }\end{array}$ & $\begin{array}{l}\text { USA } \\
\text { Dascena Inc., University of } \\
\text { California \& Stanford University }\end{array}$ & 68,319 & NR & $\mathrm{ICU}$ \\
\hline Nemati 2018 & $\begin{array}{l}\text { Retrospective time } \\
\text { series } \\
\text { multi-center ( } 3 \\
\text { sites) }\end{array}$ & $\begin{array}{l}\text { USA, Georgia } \\
\text { Emory University School of } \\
\text { Medicine \& Georgia Institute of } \\
\text { Technology }\end{array}$ & 69,938 & NR & $\mathrm{ICU}$ \\
\hline Parreco 2018 & $\begin{array}{l}\text { Retrospective time } \\
\text { series } \\
\text { single center }\end{array}$ & $\begin{array}{l}\text { USA, Florida } \\
\text { University of Miami }\end{array}$ & NA $(22,201)$ & NA & $\mathrm{ICU}$ \\
\hline Taneja 2017 & $\begin{array}{l}\text { Prospective case } \\
\text { series } \\
\text { single center }\end{array}$ & $\begin{array}{l}\text { USA, Illinois } \\
\text { University of Illinois }\end{array}$ & 444 & Suspected sepsis & NR \\
\hline Weller 2018 & $\begin{array}{l}\text { Retrospective case } \\
\text { series } \\
\text { single center }\end{array}$ & $\begin{array}{l}\text { USA, Minnesota } \\
\text { Mayo Clinic Rochester }\end{array}$ & 1,283 & Colorectal surgery patients & Surgery \\
\hline Wiens 2014 & $\begin{array}{l}\text { Retrospective case } \\
\text { series } \\
\text { single center }\end{array}$ & $\begin{array}{l}\text { USA } \\
\text { single center not specified }\end{array}$ & NR $(69,568)$ & NR & various \\
\hline
\end{tabular}

NA: Not applicable. NR: Not reported. USA: United States of America. UK: United Kingdom. ICU: Intensive care unit. ED: Emergency department. 
included sepsis $^{33,35,72,78,81,85,93,94}$, acute kidney injury ${ }^{70,78-80,82,84,91}$, surgical site infections ${ }^{33,73,76,87,92}$, central line-associated bloodstream infections ${ }^{77,86}$, Clostridium difficile ${ }^{83,88}$, pulmonary aspergillosis $^{89}$, bacteremia ${ }^{90}$, fibrosis ${ }^{71}$, urine tract infection ${ }^{33,74}$ and infections in general ${ }^{75}$.

Almost half of the studies compared different machine learning algorithms, while the others focused only on Bayesian algorithms ${ }^{73,92}$, decision tree algorithms ${ }^{84}$, ensemble algorithms $\mathrm{s}^{35,71,82,83,90,93}$, regression algorithms $\mathrm{s}^{33,78,85}$, regularization algorithms $\mathrm{s}^{81,88}$ and rule learning ${ }^{70}$. The most frequently applied model was random forest (15 studies) followed by logistic regression (10 studies), support vector machines (5 studies), naïve Bayes ( 5 studies) and gradient tree boosting (5 studies).

One study compared three different sampling methods for handling class imbalance; under-sampling the majority class (RANDu), over-sampling the minority class (RANDo) and synthetic minority over-sampling (SMOTE). This was a very large study including more than 500,000 patients to predict the onset of infections ${ }^{75}$. The authors found that SMOTE outperformed the other techniques and improved model sensitivity. Two other very large studies used the RANDu method ${ }^{80}$ and mini-batch stochastic gradient descent with backpropagation ${ }^{85}$. No other studies were concerned with imbalance in disease positive and negative classification.

Machine learning models were developed and validated in 26 studies and subsequently tested in an independent dataset in four studies $^{35,72,75,77}$.

The machine learning algorithms used are illustrated in Table 10.

Outcome measures. The most frequently reported outcome measure was the ROC AUC. Three studies did not report this measure: Ahmed et al. $2015^{70}$ developed an algorithm based on decision rules; Legrand et al. $2013^{91}$ was primarily interested in identifying risk factors of AKI after cardiac surgery; and Scicluna et al. $2017^{93}$ was primarily concerned with identifying genetic biomarkers of sepsis.

Sensitivity and specificity were reported together in 14 studies $^{35,70-72,74,75,78,81-84,86,90,92}$. When specificity was not reported, sensitivity was reported together with PPV; and when sensitivity was not reported, this was due to sensitivity being set at a fixed value to report other diagnostic performance measures. In relation to the prior observation, more studies reported PPV than NPV. Four studies reporting likelihood ratios reported both negative and positive likelihood ratios ${ }^{70,74,81,84}$.

An overview of measured outcomes is illustrated in Table 11.

Ongoing studies. Four trials are currently ongoing, one in Germany and the others in the USA, all concerned with the prediction of sepsis. Three of them are prospective studies and one is retrospective. The retrospective study aims to develop a prediction algorithm based on claims data, EHRs, risk factors and survey data of an estimated 50,000 adult patients admitted to the ED. The German study NCT03661450 95 is a single-arm trial evaluating the utility of a CDS system to identify SIRS or sepsis from EHRs in a pediatric ICU population. Another single-arm trial NCT03655626 $6^{47}$ is concerned with implementing a sepsis prediction algorithm in clinical practice as an early warning system. NCT03644940 46 is comparing two versions of InSight introduced into clinical practice as an early warning system.

\section{Discussion and conclusions}

This systematic literature review shows that over the last 2 decades, there has been an increased interest in CDS as means of supporting clinicians in acute care. CDS has been investigated for several applications ranging from the detection of health conditions $^{60,61}$, to the prediction of deterioration or adverse events $^{40,55,76,81,83,84}$. Applications also include therapy guidance, as well as updating clinicians on new or changed recommendations ${ }^{96}$. CDS can also provide guidance by predicting clinical trajectories for different patient profiles over time ${ }^{97}$.

From rule-based algorithms and simple regression models, CDS has evolved to encompass a multitude of techniques in Machine-Learning ${ }^{98}$. These techniques can be dependent on the problem selected and the data types used. Across the three disease areas investigated, the frequent use of random forest classifiers $(28.1 \%)$, support vector machines $(21.9 \%)$, boosting techniques (20.3\%), LASSO regression (18.8\%) and unspecified logistic regression models (10.9\%) were observed. The use of more complex modeling such as maximum entropy, Hidden Markov Models (for temporal data analysis) as well as Convolutional Neural Networks has also emerged over the last few years. In the respiratory distress area, the use of NLP models is more common as radiology reports and clinical notes are the main source of input. Different image analysis techniques have been developed to aid in the prediction and diagnosis of respiratory events from radiology images.

Typical measures of NLP model performance include sensitivity, specificity and predictive values. In measuring $\mathrm{ML}$ algorithm performance, sensitivity, specificity and ROC AUC are more common. A wide range of outcome measure were reported in research on less-investigated health conditions ${ }^{40,67}$; and also when uncommon, more complex algorithms were compared to basic algorithms $\mathrm{s}^{74,78,81,84}$. This is not surprising given the novelty of these applications.

Many of the ML algorithms and all of the NLP models covered in this work were based on medical data collected in certain clinical sites rather than publicly available data. Datasets from national audits, completed studies or other online sources can additionally play a role, particularly in model validation and testing. This could aid in the adoption and wider use of CDS systems. In this SLR, publicly available datasets were mainly utilized for developing prediction models of heart arrhythmias ${ }^{29-31}$, hypotension ${ }^{32}$, septic shock $^{28,33,40,41}, \mathrm{COPD}^{50}$, pneumonia ${ }^{33}$ and

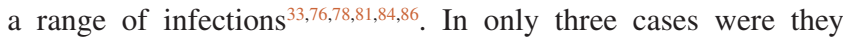
used for testing model performance in sepsis and septic shock prediction; this included the Insight algorithm ${ }^{35,85,93}$. 


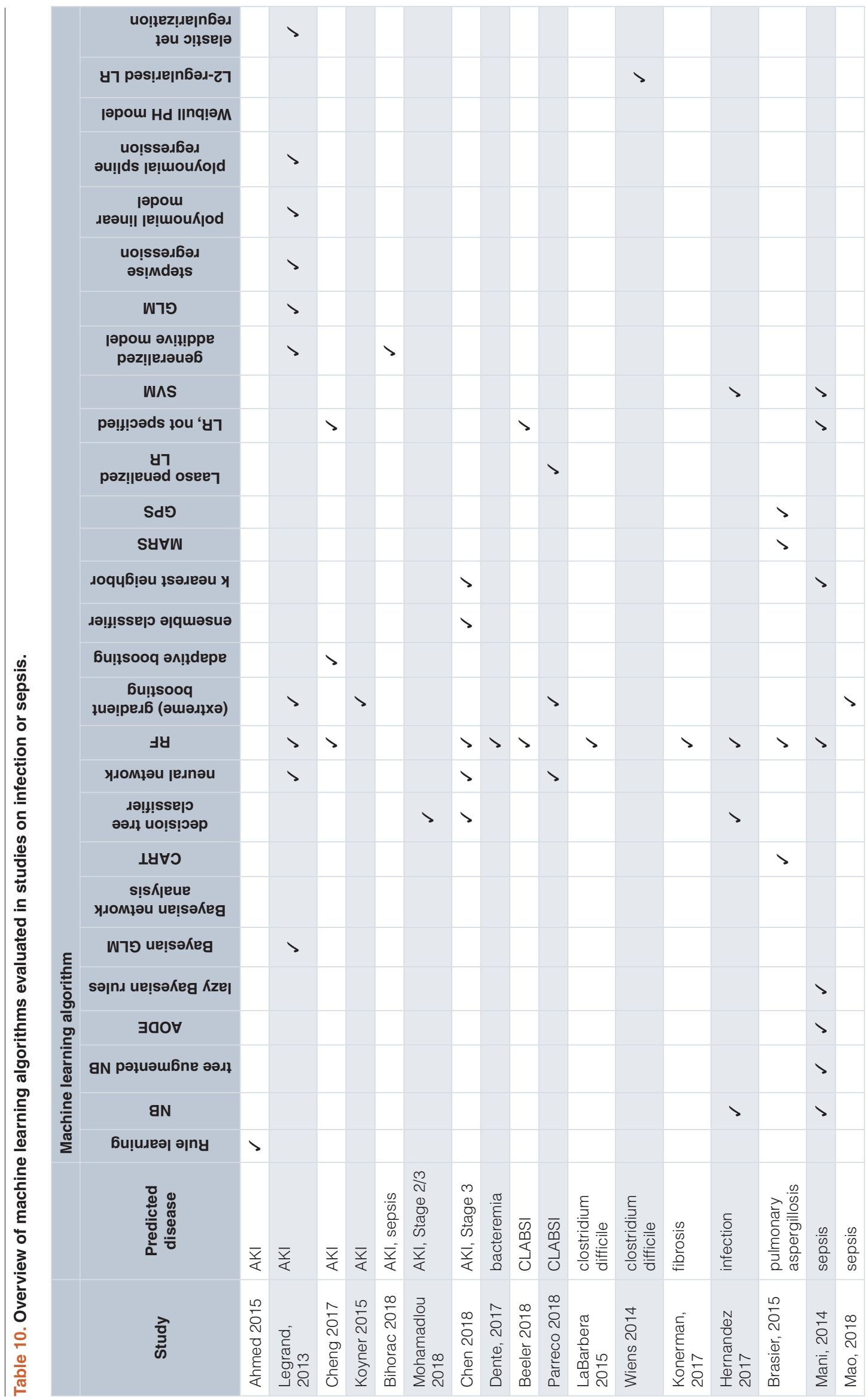




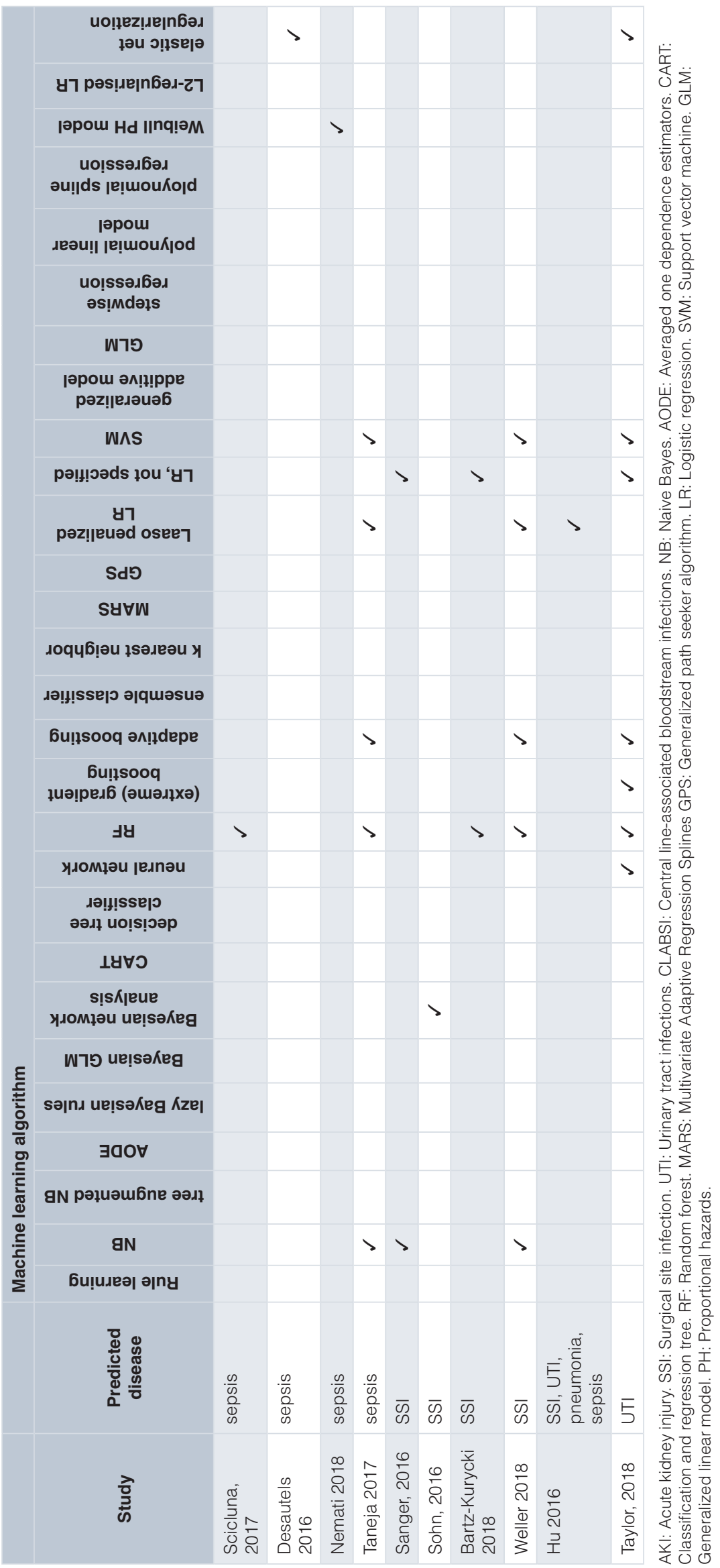




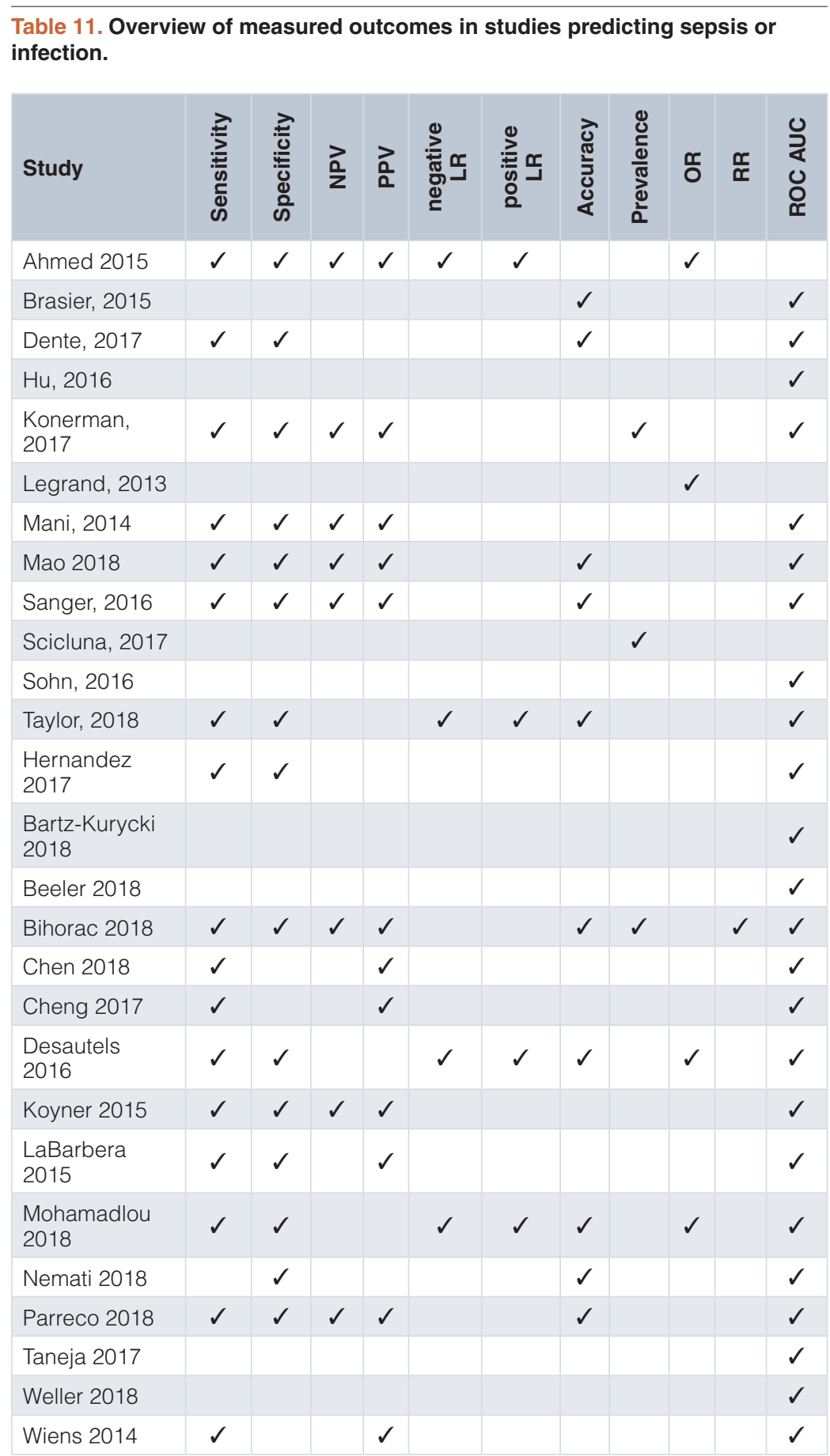

NPV: Negative predictive value. PPV: Positive predictive value. LR: Likelihood ratio. OR: Odds ratio, RR: Risks ratio. ROC AUC: Receiver operator curve area under the curve.

Most of the studies identified in this SLR were retrospective and originated in the USA where electronic health records (EHR) are commonly used. This makes it easier to access and compile large amounts of patient-level information. Many of the studies on shock and infection/sepsis based their models on data extracted from EHRs and utilized large sample sizes. The diversity in the identified CDS systems makes it challenging to draw conclusions on methodology. The lack of comparisons between different classifiers within studies, especially for the indication of shock, adds to this challenge. To assess the effectiveness of ML algorithms, future research should evaluate multiple algorithms on standard well-labeled datasets. 
Class imbalance can be an important issue when training classifiers on datasets for the conditions highlighted in this work. Unequal distributions can arise naturally between disease negative and positive classes when forming validation sets, particularly when disease prevalence is $\operatorname{low}^{75}$. We refer the reader to several machine learning reviews that have addressed this issue ${ }^{99-101}$. Another important issue in forming disease positive classes relates to the analysis of repeated-measures within subjects, for example, when clinical records are available for each hospitalization day. Several studies have approached this by selecting the first record indicating positive for a health condition. Few researchers have utilized all records and corrected for withinsubject variation. An example is the selection of cases depending on observed correlation decay ${ }^{52}$.

In all three areas investigated, the number of retrospective studies exceeded by far the number of prospective studies conducted in a clinical setting. This highlights the challenges in substantiating clinical performance while bringing new clinical decision tools to routine in-hospital patientcare. Examples of algorithms that can be integrated in clinical practice include InSight ${ }^{45,46}$ and Sepsis Watch ${ }^{47}$ which are intended for predicting sepsis and septic shock.

The current systematic literature review did not search multiple bibliographic databases or clinical trial registers; and focused on diagnostic performance rather than other outcomes. In fact, during study screening, trials that evaluated the impact of early warning systems on measures of clinical workflow, rate of re-admissions and/or mortality were discarded as they are somehow out of the focus of this work. This implies that there may be more CDS systems used in practice for the three populations investigated within this research, where the outcomes measured are different. Limiting the search to publications in English and to studies conducted in particular countries may have further limited the studies selected. Nevertheless, studies identified within each population represented a diverse range of models applied in different hospital settings trained to predict a range of health conditions. The most widely researched conditions were sepsis and septic shock, venous thromboembolisms, acute kidney injury and surgical site infections.

Specific challenges were identified in collecting sufficient data for training CDS systems on hemodynamic instability. Patients who are, for example, at risk of hemorrhage due to a traumatic injury need to be carefully monitored; and the speed by which they reach a critical state may influence data and study management. It may also be difficult to find healthy volunteers who are willing to undergo procedures like lower body negative pressure which can be unpleasant ${ }^{36}$. Identification of cases in need of hemodynamic interventions can lend towards larger sample size $^{19}$. Other conditions that need further attention are clostridium difficile and CLABSI. Prediction models were driven by almost perfect specificity and very low $(<10 \%)$ sensitivity ${ }^{77,83,86,88}$. Considering that these studies used a wide range of features from the EHRs and a large number of patients, except LaBarbera,
Nikiforov $^{83}$, there is a need to better understand the risk factors to improve sensitivity.

Based on the literature reviewed in this work, as well as several recent surveys and workshops, we would recommend the following points to be addressed when bringing a new CDS tool to critical care ${ }^{14,102-104}$ :

- Integrating CDS in clinical workflows without adding unnecessary extra work to busy clinical teams. The CDS101 toolbox by HIMMS highlights the "CDS five rights", which are certainly applicable to critical care ${ }^{105}$ : Providing the right information in the right intervention format, to the right person at the right point in their workflow, and through the right channel.

- Developing tools and concrete proof-points able to assess CDS efficacy in the clinic. This also highlights the importance of providing continuous feedback to clinicians.

- The importance of easy to use user interfaces and focusing on human-computer interaction during deployment.

- Efficient training that is available when needed.

- Being aware of alert or alarm fatigue and not overloading clinicians with alerts due to CDS. The intensive care unit is already plagued with alarms, and if anything, CDS should help in reducing alarms by bundling alerts according to underlying conditions.

- Displaying the rationale for decisions as well as the underlying data to clinical users would lead to improved adoption.

- Understanding ethical challenges for CDS, as well as a careful risk assessment in every site before deployment ${ }^{106}$.

- Being able to repeat/standardize implementation across organizations - most prospective studies reviewed in this work covered single centers. Only a few were multicenter studies.

\section{Data availability}

\section{Underlying data}

All data underlying the results are available as part of the article and no additional source data are required

\section{Extended data}

Figshare: Evidence-based Clinical Decision Support Systems for the prediction and detection of three disease states in critical care: A systematic literature review. Extended data - Table 1-Search strategy for shock (hemodynamic (in-stability) in MEDLINE. docx. https://doi.org/10.6084/m9.figshare.9892109.v1 $1^{25}$.

Figshare: Working title: Evidence-based Clinical Decision Support Systems for the prediction and detection of three disease states in critical care: A systematic literature review. Extended data - Table 2-Search strategy for respiratory distress or respiratory failure in MEDLINE.docx. https://doi.org/10.6084/ m9.figshare.9892112.v1 $1^{26}$. 
Figshare: Working title: Evidence-based Clinical Decision Support Systems for the prediction and detection of three disease states in critical care: A systematic literature review. Extended data - Table 3-Search strategy for infection or sepsis in MEDLINE. docx. https://doi.org/10.6084/m9.figshare.9892115.v127.

\section{Reporting guidelines}

Figshare: PRISMA checklist for 'Evidence-based Clinical Decision Support Systems for the prediction and detection of three disease states in critical care: A systematic literature review'. https://doi.org/10.6084/m9.figshare.9894107.v1 ${ }^{107}$.
Data are available under the terms of the Creative Commons Zero "No rights reserved" data waiver (CC0 1.0 Public domain dedication).

\section{Acknowledgments}

We would like to thank Mark Connolly from Global Market Access Solutions Sàrl for his contribution during the whole project.
1. Molina JA, Seow E, Heng BH, et al:: Outcomes of direct and indirect medical intensive care unit admissions from the emergency department of an acute care hospital: a retrospective cohort study. BMJ Open. 2014; 4(11): e005553. PubMed Abstract | Publisher Full Text | Free Full Text

2. Winters B, Custer J, Galvagno SM Jr, et al.: Diagnostic errors in the intensive care unit: a systematic review of autopsy studies. BMJ Qual Saf. 2012; 21(11): 894-902.

PubMed Abstract | Publisher Full Text

3. Rothschild JM, Landrigan CP, Cronin JW, et al:: The Critical Care Safety Study: The incidence and nature of adverse events and serious medical errors in intensive care. Crit Care Med. 2005; 33(8): 1694-700.

PubMed Abstract | Publisher Full Text

4. Donovan JL, Kanaan AO, Thomson MS, et al.: Effect of clinical decision support on psychotropic medication prescribing in the long-term care setting. $J A m$ Geriatr Soc. 2010; 58(5): 1005-7.

PubMed Abstract | Publisher Full Text

5. Field TS, Rochon P, Lee M, et al.: Computerized clinical decision support during medication ordering for long-term care residents with renal insufficiency. $\mathrm{J} \mathrm{Am}$ Med Inform Assoc. 2009; 16(4): 480-5.

PubMed Abstract | Publisher Full Text | Free Full Text

6. Kennedy CC, Campbell G, Garg AX, et al:: Piloting a renal drug alert system for prescribing to residents in long-term care. J Am Geriatr Soc. 2011; 59(9): 1757-9.

PubMed Abstract | Publisher Full Text | Free Full Text

7. Tamblyn R, Eguale T, Buckeridge DL, et al.: The effectiveness of a new generation of computerized drug alerts in reducing the risk of injury from drug side effects: a cluster randomized trial. J Am Med Inform Assoc. 2012; 19(4): 635-43.

PubMed Abstract | Publisher Full Text | Free Full Text

8. Marasinghe KM: Computerised clinical decision support systems to improve medication safety in long-term care homes: a systematic review. BMJ Open. 2015; 5(5): e006539.

PubMed Abstract | Publisher Full Text | Free Full Text

9. Quinn CC, Clough SS, Minor JM, et al:: WellDoc mobile diabetes management randomized controlled trial: change in clinical and behavioral outcomes and patient and physician satisfaction. Diabetes Technol Ther. 2008; 10(3): 160-8. PubMed Abstract | Publisher Full Text

10. Coiera $\mathrm{E}$, Lau $\mathrm{AY}$, Tsafnat $\mathrm{G}$, et al.: The changing nature of clinical decision support systems: a focus on consumers, genomics, public health and decision safety. Yearb Med Inform. 2009; 84-95. PubMed Abstract | Publisher Full Text

11. Agoritsas $\mathrm{T}$, Heen $\mathrm{AF}$, Brandt $\mathrm{L}$, et al.: Decision aids that really promote shared decision making: the pace quickens. BMJ. 2015; 350: g7624.

PubMed Abstract | Publisher Full Text | Free Full Text

12. Vincent JL, Einav $\mathrm{S}$, Pearse $\mathrm{R}$, et al:: Improving detection of patient deterioration in the general hospital ward environment. Eur J Anaesthesiol. 2018; 35(5): $325-333$.

PubMed Abstract | Free Full Text

13. Cox JC, Sadiraj V, Schnier KE, et al:: Higher Quality and Lower Cost from Improving Hospital Discharge Decision Making. J Econ Behav Organ. 2016; 131(B): 1-16.

PubMed Abstract | Publisher Full Text | Free Full Text

14. Tcheng JE, Bakken S, Bates DW, et al:: Optimizing Strategies for Clinical Decision Support. In The Learning Health System Series, N.A.o. Medicine, Editor. 2017: Washington DC USA.

Reference Source

15. Duncan H, Hutchison J, Parshuram CS: The Pediatric Early Warning System score: a severity of illness score to predict urgent medical need in hospitalized children. J Crit Care. 2006; 21(3): 271-8.

PubMed Abstract | Publisher Full Text

16. Parshuram C, Duncan HP, Joffe AR, et al.: Multicentre validation of the bedside paediatric early warning system score: a severity of illness score to detect evolving critical illness in hospitalised children. Crit Care. 2011; 15(4): R184. PubMed Abstract | Publisher Full Text | Free Full Text

17. Chapman SM, Wray J, Oulton K, et al:: 'The Score Matters': wide variations in predictive performance of 18 paediatric track and trigger systems. Arch Dis Child. 2017; 102(6): 487-495.

PubMed Abstract | Publisher Full Text

18. Philips. [Accessed 2nd July 2018]. 2018. Reference Source

19. Potes C, Conroy B, Xu-Wilson M, et al.: A clinical prediction model to identify patients at high risk of hemodynamic instability in the pediatric intensive care unit. Crit Care. 2017; 21(1): 282

PubMed Abstract | Publisher Full Text | Free Full Text

20. Hravnak M, Devita MA, Clontz A, et al:: Cardiorespiratory instability before and after implementing an integrated monitoring system. Crit Care Med. 2011 39(1): 65-72.

PubMed Abstract | Publisher Full Text | Free Full Text

21. Gaieski DF, Mikkelsen ME, Band RA, et al:: Impact of time to antibiotics on survival in patients with severe sepsis or septic shock in whom early goaldirected therapy was initiated in the emergency department. Crit Care Med. 2010; 38(4): 1045-53.

PubMed Abstract | Publisher Full Text

22. Critical care statistics. [cited 2019 September 10] Reference Source

23. Mayr FB, Yende S, Angus DC: Epidemiology of severe sepsis. Virulence. 2014; 5(1): 4-11.

PubMed Abstract | Publisher Full Text | Free Full Text

24. Sakr Y, Jaschinski U, Wittebole X, et al.: Sepsis in Intensive Care Unit Patients: Worldwide Data From the Intensive Care over Nations Audit. Open Forum Infect Dis. 2018; 5(12): ofy313.

PubMed Abstract | Publisher Full Text | Free Full Text

25. Medic G, Kließ MK, Atallah L, et al.: Evidence-based Clinical Decision Support Systems for the prediction and detection of three disease states in critical care: A systematic literature review. Extended data - Table 1-Search strategy for shock (hemodynamic (in-stability) in MEDLINE.docx. figshare. Dataset. for shoch.

http://www.doi.org/10.6084/m9.figshare.9892109.v1

26. Medic G, Kließ MK, Atallah L, et al: Working title: Evidence-based Clinical Decision Support Systems for the prediction and detection of three disease states in critical care: A systematic literature review. Extended data - Table 2-Search strategy for respiratory distress or respiratory failure in MEDLINE. docx. figshare. Dataset. 2019. http://www.doi.org/10.6084/m9.figshare.9892112.v1

27. Medic G, Kließ MK, Atallah L, et al:: Working title: Evidence-based Clinical Decision Support Systems for the prediction and detection of three disease states in critical care: A systematic literature review. Extended data - Table 3-Search strategy for infection or sepsis in MEDLINE.docx. figshare. Dataset. 2019. http://www.doi.org/10.6084/m9.figshare.9892115.v1

28. Ghosh S, Li J, Cao L, et al.: Septic shock prediction for ICU patients via coupled HMM walking on sequential contrast patterns. J Biomed Inform. 2017; 66 : 19-31.

PubMed Abstract | Publisher Full Text 
29. Li Q, Rajagopalan C, Clifford GD: Ventricular fibrillation and tachycardia classification using a machine learning approach. IEEE Trans Biomed Eng 2014; 61(6): 1607-13.

PubMed Abstract | Publisher Full Text

30. Ebrahimzadeh E, Kalantari M, Joulani M, et al:: Prediction of paroxysmal Atria Fibrillation: A machine learning based approach using combined feature vector and mixture of expert classification on HRV signal. Comput Methods Programs Biomed. 2018; 165: 53-67. PubMed Abstract | Publisher Full Text

31. Strodthoff $N$, Strodthoff $C$ : Detecting and interpreting myocardial infarction using fully convolutional neural networks. Physiol Meas. 2018; 40(1): 015001 PubMed Abstract | Publisher Full Text

32. Donald R, Howells T, Piper I, et al.: Forewarning of hypotensive events using a Bayesian artificial neural network in neurocritical care. J Clin Monit Comput. 2018; 33(1): 39-51

PubMed Abstract | Publisher Full Text

33. Hu Z, Melton GB, Moeller ND, et al.: Accelerating Chart Review Using Automated Methods on Electronic Health Record Data for Postoperative Complications. AMIA Annu Symp Proc. 2016; 2016: 1822-1831. PubMed Abstract | Free Full Text

34. Mahajan D, Dong Y, Saxon LA, et al.: Performance of an automatic arrhythmi classification algorithm: comparison to the ALTITUDE electrophysiologist panel adjudications. Pacing Clin Electrophysiol. 2014; 37(7): 889-99. PubMed Abstract | Publisher Full Text

35. Mao $\mathrm{Q}$, Jay $\mathrm{M}$, Hoffman $\mathrm{JL}$, et al: Multicentre validation of a sepsis prediction algorithm using only vital sign data in the emergency department, general ward and ICU. BMJ Open. 2018; 8(1): e017833.

PubMed Abstract | Publisher Full Text | Free Full Text

36. Reljin N, Zimmer G, Malyuta Y, et al:: Using support vector machines on photoplethysmographic signals to discriminate between hypovolemia and euvolemia. PLoS One. 2018; 13(3): e0195087. PubMed Abstract | Publisher Full Text | Free Full Text

37. Sideris $\mathrm{C}$, Pourhomayoun $\mathrm{M}$, Kalantarian $\mathrm{H}$, et al:: A flexible data-driven comorbidity feature extraction framework. Comput Biol Med. 2016; 73: 165-72. PubMed Abstract | Publisher Full Text

38. Blecker S, Katz SD, Horwitz LI, et al:: Comparison of Approaches for Heart Failure Case Identification From Electronic Health Record Data. JAMA Cardiol. 2016; 1(9): 1014-1020.

PubMed Abstract | Publisher Full Text | Free Full Text

39. Blecker S, Sontag D, Horwitz LI, et al:: Early Identification of Patients With Acute Decompensated Heart Failure. J Card Fail. 2018; 24(6): 357-362. PubMed Abstract | Publisher Full Text | Free Full Text

40. Calvert J, Desautels $\mathrm{T}$, Chettipally $\mathrm{U}$, et al.: High-performance detection and early prediction of septic shock for alcohol-use disorder patients. Ann Med Surg (Lond). 2016; 8: 50-5

PubMed Abstract | Publisher Full Text | Free Full Text

41. Henry K, Hager DN, Pronovost PJ, et al: A targeted real-time early warning score (TREWScore) for septic shock. Science Translational Medicine. 2015; 7(299): 299ra122

PubMed Abstract | Publisher Full Text

42. Panahiazar M, Taslimitehrani V, Pereira N, et al.: Using EHRs and Machine Learning for Heart Failure Survival Analysis. Stud Health Technol Inform. 2015; 216: 40-4

PubMed Abstract | Free Full Text

43. NCT02934971: Optimized Multi-modality Machine Learning Approach During Cardio-toxic Chemotherapy to Predict Arising Heart Failure (MERMAID). Reference Source

44. NCT03582501: Measurement of Hemodynamic Responses to Lower Body Negative Pressure (LBNP).

Reference Source

45. NCT03235193: Predictive algoRithm for EValuation and Intervention in SEpsis (PREVISE).

Reference Source

46. NCT03644940: Subpopulation-Specific Sepsis Identification Using Machine Learning

Reference Source

47. NCT03655626: Implementation and Evaluations of Sepsis Watch Reference Source

48. Bejan CA, Vanderwende L, Evans HL, et al:: On-time clinical phenotype prediction based on narrative reports. AMIA Annu Symp Proc. 2013; 2013: 103-10.

PubMed Abstract | Free Full Text

49. Kumamaru KK, George E, Aghayev A, et al.: Implementation and Performance of Automated Software for Computing Right-to-Left Ventricular Diameter Ratio From Computed Tomography Pulmonary Angiography Images. J Comput Assist Tomogr. 2016; 40(3): 387-92.

PubMed Abstract | Publisher Full Text | Free Full Text

50. Bodduluri S, Newell JD Jr, Hoffman EA, et al.: Registration-based lung mechanical analysis of chronic obstructive pulmonary disease (COPD) using a supervised machine learning framework. Acad Radiol. 2013; 20(5): 527-36. PubMed Abstract | Publisher Full Text | Free Full Text

51. Biesiada J, Chidambaran V, Wagner M, et al:: Genetic risk signatures of opioid-induced respiratory depression following pediatric tonsillectomy. Pharmacogenomics. 2014; 15(14): 1749-1762.

PubMed Abstract | Publisher Full Text | Free Full Text

52. Reamaroon N, Sjoding MW, Lin K, et al.: Accounting for Label Uncertainty in Machine Learning for Detection of Acute Respiratory Distress Syndrome. IEEE J Biomed Health Inform. 2019; 23(1): 407-415.

PubMed Abstract | Publisher Full Text | Free Full Text

53. Vinson DR, Morley JE, Huang J, et al:: The Accuracy of an Electronic Pulmonary Embolism Severity Index Auto-Populated from the Electronic Health Record: Setting the stage for computerized clinical decision support. Appl Clin Inform. 2015; 6(2): 318-33.

PubMed Abstract | Publisher Full Text | Free Full Text

54. Huesch MD, Cherian R, Labib S, et al.: Evaluating Report Text Variation and Informativeness: Natural Language Processing of CT Chest Imaging for Pulmonary Embolism. J Am Coll Radiol. 2018; 15(3 Pt B): 554-562. PubMed Abstract | Publisher Full Text

55. Mortazavi BJ, Desai N, Zhang J, et al.: Prediction of Adverse Events in Patients Undergoing Major Cardiovascular Procedures. IEEE J Biomed Health Inform. 2017; 21(6): 1719-1729.

PubMed Abstract | Publisher Full Tex

56. González G, Ash SY, Vegas-Sánchez-Ferrero G, et al.: Disease Staging and Prognosis in Smokers Using Deep Learning in Chest Computed Tomography. Am J Respir Crit Care Med. 2018; 197(2): 193-203. PubMed Abstract | Publisher Full Text | Free Full Text

57. Choi $Y$, Liu TT, Pankratz DG, et al: Identification of usual interstitial pneumonia pattern using RNA-Seq and machine learning: challenges and solutions. $B M C$ Genomics. 2018; 19(Suppl 2): 101

PubMed Abstract | Publisher Full Text | Free Full Text

58. Yu S, Kumamaru KK, George E, et al:: Classification of CT pulmonary angiography reports by presence, chronicity, and location of pulmonary embolism with natural language processing. J Biomed Inform. 2014; 52: 386-93. PubMed Abstract | Publisher Full Text | Free Full Text

59. Swartz J, Koziatek C, Theobald J, et al:: Creation of a simple natural language processing tool to support an imaging utilization quality dashboard. Int $\mathrm{J}$ Med Inform. 2017; 101: 93-99.

PubMed Abstract | Publisher Full Text

60. Liu V, Clark MP, Mendoza M, et al:: Automated identification of pneumonia in chest radiograph reports in critically ill patients. BMC Med Inform Decis Mak. 2013; 13: 90.

PubMed Abstract | Publisher Full Text | Free Full Text

61. Haug PJ, Ferraro JP, Holmen J, et al:: An ontology-driven, diagnostic modeling system. J Am Med Inform Assoc. 2013; 20(e1): e102-10.

PubMed Abstract | Publisher Full Text | Free Full Text

62. Dublin S, Baldwin E, Walker RL, et al:: Natural Language Processing to identify pneumonia from radiology reports. Pharmacoepidemiol Drug Saf. 2013; 22(8): 834-41.

PubMed Abstract | Publisher Full Text | Free Full Text

63. Jones BE, South BR, Shao Y, et al:: Development and Validation of a Natural Language Processing Tool to Identify Patients Treated for Pneumonia across VA Emergency Departments. Appl Clin Inform. 2018; 9(1): 122-128. PubMed Abstract | Publisher Full Text | Free Full Text

64. Rochefort CM, Verma AD, Eguale T, et al:: A novel method of adverse event detection can accurately identify venous thromboembolisms (VTEs) from narrative electronic health record data. J Am Med Inform Assoc. 2015; 22(1): 155-65.

PubMed Abstract | Publisher Full Text | Free Full Text

65. Tian Z, Sun S, Eguale T, et al:: Automated Extraction of VTE Events From Narrative Radiology Reports in Electronic Health Records: A Validation Study. Med Care. 2017; 55(10): e73-e80.

PubMed Abstract | Publisher Full Text | Free Full Text

66. Pham AD, Névéol A, Lavergne $T$, et al:: Natural language processing of radiology reports for the detection of thromboembolic diseases and clinically relevant incidental findings. BMC Bioinformatics. 2014; 15: 266.

PubMed Abstract | Publisher Full Text | Free Full Text

67. Silva S, Ait Aissa D, Cocquet $P$, et al.: Combined Thoracic Ultrasound Assessment during a Successful Weaning Trial Predicts Postextubation Distress. Anesthesiology. 2017; 127(4): 666-674. PubMled Abstract | Publisher Full Text

68. Phillips C, Mac Parthaláin N, Syed Y, et al.: Short-Term Intra-Subject Variation in Exhaled Volatile Organic Compounds (VOCs) in COPD Patients and Healthy Controls and Its Effect on Disease Classification. Metabolites. 2014; 4(2): 300-18.

PubMed Abstract | Publisher Full Text | Free Full Text

69. Phillips $\mathrm{R}$, Williams $\mathrm{D}$, Bowen $\mathrm{D}$, et al:: Reaching a consensus on research priorities for supporting women with autoimmune rheumatic diseases during pre-conception, pregnancy and early parenting: A Nominal Group Technique exercise with lay and professional stakeholders. Wellcome Open Res. 2018; 3 75.

PubMed Abstract | Publisher Full Text | Free Full Text

70. Ahmed A, Vairavan S, Akhoundi A, et al.: Development and validation of electronic surveillance tool for acute kidney injury: A retrospective analysis. J Crit Care. 2015; 30(5): 988-93. PubMed Abstract | Publisher Full Text 
71. Konerman MA, Lu D, Zhang $\mathrm{Y}$, et al:: Assessing risk of fibrosis progression and liver-related clinical outcomes among patients with both early stage and advanced chronic hepatitis C. PLoS One. 2017; 12(11): e0187344. PubMed Abstract | Publisher Full Text | Free Full Text

72. Mani S, Ozdas A, Aliferis C, et al.: Medical decision support using machine learning for early detection of late-onset neonatal sepsis. J Am Med Inform Assoc. 2014; 21(2): 326-36.

PubMed Abstract | Publisher Full Text | Free Full Text

73. Sohn S, Larson DW, Habermann EB, et al.: Detection of clinically important colorectal surgical site infection using Bayesian network. J Surg Res. 2017; 209: 168-173.

PubMed Abstract | Publisher Full Text | Free Full Text

74. Taylor RA, Moore CL, Cheung $\mathrm{KH}$, et al:: Predicting urinary tract infections in the emergency department with machine learning. PLoS One. 2018; 13(3): e0194085.

PubMed Abstract | Publisher Full Text | Free Full Text

75. Hernandez B, Herrero $P$, Rawson TM, et al:: Supervised learning for infection risk inference using pathology data. BMC Med Inform Decis Mak. 2017; 17(1): 168.

PubMed Abstract | Publisher Full Text | Free Full Text

76. Bartz-Kurycki MA, Green C, Anderson KT, et al.: Enhanced neonatal surgical site infection prediction model utilizing statistically and clinically significant variables in combination with a machine learning algorithm. Am J Surg. 2018; 216(4): 764-777.

PubMed Abstract | Publisher Full Text

77. Beeler C, Dbeibo L, Kelley K, et al:: Assessing patient risk of central lineassociated bacteremia via machine learning. Am J Infect Control. 2018; 46(9): 986-991.

PubMed Abstract | Publisher Full Text

78. Bihorac A, Ozrazgat-Baslanti T, Ebadi A, et al:: MySurgeryRisk: Development and Validation of a Machine-learning Risk Algorithm for Major Complications and Death After Surgery. Ann Surg. 2019; 269(4): 652-662. PubMed Abstract | Publisher Full Text | Free Full Text

79. Chen W, Hu Y, Zhang X, et al.: Causal risk factor discovery for severe acute kidney injury using electronic health records. BMC Med Inform Decis Mak. 2018; 18(Suppl 1): 13.

PubMed Abstract | Publisher Full Text | Free Full Text

80. Cheng P, Waitman LR, Hu Y, et al:: Predicting Inpatient Acute Kidney Injury over Different Time Horizons: How Early and Accurate? AMIA Annu Symp Proc 2018; 2017: 565-574.

PubMed Abstract | Free Full Text

81. Desautels $\mathrm{T}$, Calvert J, Hoffman J, et al:: Prediction of Sepsis in the Intensive Care Unit With Minimal Electronic Health Record Data: A Machine Learning Approach. JMIR Med Inform. 2016; 4(3): e28.

PubMed Abstract | Publisher Full Text | Free Full Text

82. Koyner JL, Carey KA, Edelson DP, et al.: The Development of a Machine Learning Inpatient Acute Kidney Injury Prediction Model. Crit Care Med. 2018 46(7): 1070-1077.

PubMed Abstract | Publisher Full Text

83. LaBarbera FD, Nikiforov I, Parvathenani A, et al:: A prediction model for Clostridium difficile recurrence. J Community Hosp Intern Med Perspect. 2015; 5(1): 26033

PubMed Abstract | Publisher Full Text | Free Full Text

84. Mohamadlou H, Lynn-Palevsky A, Barton C, et al:: Prediction of Acute Kidney Injury With a Machine Learning Algorithm Using Electronic Health Record Data. Can J Kidney Health Dis. 2018; 5: 2054358118776326.

PubMed Abstract | Publisher Full Text | Free Full Text

85. Nemati S, Holder A, Razmi F, et al:: An Interpretable Machine Learning Model for Accurate Prediction of Sepsis in the ICU. Crit Care Med. 2018; 46(4): 547-553. PubMed Abstract | Publisher Full Text | Free Full Text

86. Parreco JP, Hidalgo AE, Badilla AD, et al:: Predicting central line-associated bloodstream infections and mortality using supervised machine learning. J Crit Care. 2018; 45: 156-162.

PubMed Abstract | Publisher Full Text

87. Weller GB, Lovely J, Larson DW, et al.: Leveraging electronic health records for predictive modeling of post-surgical complications. Stat Methods Med Res. 2018; 27(11): 3271-3285

PubMed Abstract | Publisher Full Text

88. Wiens J, Campbell WN, Franklin ES, et al: Learning Data-Driven Patient Risk
Stratification Models for Clostridium difficile. Open Forum Infect Dis. 2014; 1(2): ofu045.

PubMed Abstract | Publisher Full Text | Free Full Text

89. Brasier AR, Zhao Y, Spratt HM, et al.: Improved Detection of Invasive Pulmonary Aspergillosis Arising during Leukemia Treatment Using a Panel of Host Response Proteins and Fungal Antigens. PLoS One. 2015; 10(11): e0143165. PubMed Abstract | Publisher Full Text | Free Full Text

90. Dente CJ, Bradley M, Schobel S, et al.: Towards precision medicine: Accurate predictive modeling of infectious complications in combat casualties. J Trauma Acute Care Surg. 2017; 83(4): 609-616.

PubMed Abstract | Publisher Full Text

91. Legrand M, Pirracchio R, Rosa A, et al:: Incidence, risk factors and prediction of post-operative acute kidney injury following cardiac surgery for active infective endocarditis: an observational study. Crit Care. 2013; 17(5): R220. PubMed Abstract | Publisher Full Text | Free Full Text

92. Sanger PC, van Ramshorst GH, Mercan E, et al.: A Prognostic Model of Surgical Site Infection Using Daily Clinical Wound Assessment. J Am Coll Surg. 2016 223(2): 259-270.e2.

PubMed Abstract | Publisher Full Text | Free Full Text

93. Scicluna BP, van Vught LA, Zwinderman AH, et al:: Classification of patients with sepsis according to blood genomic endotype: a prospective cohort study. Lancet Respir Med. 2017; 5(10): 816-826.

PubMed Abstract | Publisher Full Text

94. Taneja I, Reddy B, Damhorst G, et al:: Combining Biomarkers with EMR Data to Identify Patients in Different Phases of Sepsis. Sci Rep. 2017; 7(1): 10800. PubMed Abstract | Publisher Full Text | Free Full Text

95. NCT03661450: Evaluation of the Accuracy of a Clinical Decision-Support System (CDSS) to Support Detection of SIRS and Sepsis in Paediatric Intensive Care Patients Compared to Medical Specialists. Reference Source

96. Van de Velde S, Kortteisto T, Spitaels D, et al:: Development of a Tailored Intervention With Computerized Clinical Decision Support to Improve Quality of Care for Patients With Knee Osteoarthritis: Multi-Method Study. JMIR Res Protoc. 2018; 7(6): e154.

PubMed Abstract | Publisher Full Text | Free Full Text

97. Pinaire J, Azé J, Bringay S, et al:: Patient healthcare trajectory. An essential monitoring tool: a systematic review. Health Inf Sci Syst. 2017; 5(1): 1. PubMed Abstract | Publisher Full Text | Free Full Text

98. Middleton B, Sittig DF, Wright A: Clinical Decision Support: a 25 Year Retrospective and a 25 Year Vision. Yearb Med Inform. 2016; (Suppl 1): S103-16. PubMed Abstract | Publisher Full Text | Free Full Text

99. Longadge R, Dongre S: Class Imbalance Problem in Data Mining Review. arXiv e-prints. 2013. Reference Source

100. Buda M, Maki A, Mazurowski MA: A systematic study of the class imbalance problem in convolutional neural networks. Neural Netw. 2018; 106: 249-259. PubMed Abstract | Publisher Full Text

101. Nanni L, Fantozzi C, Lazzarini N: Coupling different methods for overcoming the class imbalance problem. Neurocomputing. 2015; 158: 48-61. Publisher Full Text

102. Kindle RD, Badawi O, Celi LA, et al.: Intensive Care Unit Telemedicine in the Era of Big Data, Artificial Intelligence, and Computer Clinical Decision Support Systems. Crit Care Clin. 2019; 35(3): 483-495. PubMed Abstract | Publisher Full Text

103. Rumsfeld JS, Joynt KE, Maddox TM: Big data analytics to improve cardiovascular care: promise and challenges. Nat Rev Cardiol. 2016; 13(6): $350-9$.

PubMed Abstract | Publisher Full Text

104. (NQF), NQF: Driving Quality and Performance Measurement-A Foundation for Clinical Decision Support: A Consensus Report. 2010; NQF: Washington, DC. Reference Source

105. HIMMS: Clinical Decision Support 101. 2019; [cited 2019]. Reference Source

106. Char DS, Shah NH, Magnus D: Implementing Machine Learning in Health Care - Addressing Ethical Challenges. N Engl J Med. 2018; 378(11): 981-983. PubMed Abstract | Publisher Full Text | Free Full Text

107. Medic G, Kließ MK, Atallah L, et al.: Evidence-based Clinical Decision Support Systems for the prediction and detection of three disease states in critical care: A systematic literature review. PRISMA Checklist. figshare. Dataset. 2019. http://www.doi.org/10.6084/m9.figshare.9894107.v1 


\section{Open Peer Review}

\section{Current Peer Review Status:}

\section{Version 1}

Reviewer Report 18 November 2019

https://doi.org/10.5256/f1000research.22530.r56200

(C) 2019 Kovacevic M. This is an open access peer review report distributed under the terms of the Creative Commons Attribution License, which permits unrestricted use, distribution, and reproduction in any medium, provided the original work is properly cited.

\section{Milena Kovacevic}

Department of Pharmacokinetics and Clinical Pharmacy, Faculty of Pharmacy, University of Belgrade, Belgrade, Serbia

The review summarizes the utilization of clinical decision support (CDS) systems in three selected states in critical care - shock/hemodynamic (in-)stability; respiratory distress/failure; and infection/sepsis. The background of the study has a strong rationale.

The study comprised the results from primary sources, describing models/algorithms used to detect and alert clinicians to the presence of these conditions, as well as models/algorithms developed to predict deterioration in an individual patient state, leading to these selected conditions.

The systematic review was performed and the findings are presented in line with the PRISMA guidelines. Variables for which data were sought were clearly stated (PICOS) in Table 1.

Specific comments:

What I found especially beneficial for the readers and future research in this area, is Table 2 with the presented collected data used for training algorithms.

It would be beneficial to provide additional information whether an internal or external validation was performed - within Table 4 (measured outcomes in studies on shock), Table 8 (measured outcomes in studies on respiratory distress/failure) and Table 11 (measured outcomes in studies on infection/sepsis).

What was the rationale for including the studies predicting acute kidney injury within the Infection/sepsis results section? If it is about the decline in glomerular filtration rate due to hypotension seen in sepsis, it might have been presented within the Shock section.

Table 7: include the abbreviations for ARDS (Acute respiratory distress syndrome), ARDE (Acute respiratory disease events) and DVT (deep vein thrombosis) below the Table. 
Table 9: include the abbreviation for AKI (Acute kidney injury) below the Table.

Are the rationale for, and objectives of, the Systematic Review clearly stated?

Yes

Are sufficient details of the methods and analysis provided to allow replication by others? Yes

Is the statistical analysis and its interpretation appropriate?

Not applicable

Are the conclusions drawn adequately supported by the results presented in the review? Yes

Competing Interests: No competing interests were disclosed.

Reviewer Expertise: Pharmacokinetics and Clinical Pharmacy; Patient outcomes.

I confirm that I have read this submission and believe that I have an appropriate level of expertise to confirm that it is of an acceptable scientific standard.

Reviewer Report 06 November 2019

https://doi.org/10.5256/f1000research.22530.r56199

(C) 2019 Nikolakopoulos S. This is an open access peer review report distributed under the terms of the Creative Commons Attribution License, which permits unrestricted use, distribution, and reproduction in any medium, provided the original work is properly cited.

\section{Stavros Nikolakopoulos}

Department of Biostatistics \& Research Support, Julius Center for Health Sciences and Primary Care, University Medical Center Utrecht, Utrecht, The Netherlands

The authors report on a systematic review in order to assess the state-of-the -art in the field of Clinical Decision Support (CDS) systems in the last 5 years (2013-2018). They review and report on study designs, outcomes and methods employed in CDS in the scientific literature as well as in study databases (like Clinicaltrials.gov).

The paper is clearly written and organized. The methodology for the systematic review is solid and comprehensive. The topic is also very relevant and timely. I do have some concerns which are mentioned below:

The authors could potentially include in the study (as described by the inclusion criteria), conference abstracts that were published only as abstracts in 2017 or 2018, even without subsequent publication. I assume they do that in order to somehow keep up with later developments even if they are not published elsewhere, given the very fast pace of the 
research area. However, they exclude protocols of studies that were published in the same (or more extended) time frame, which seems slightly inconsistent. Some discussion concerning this choice would be enlightening.

There seems to be some confusion with terminology, with unknown consequences on the review's results. The authors seem to separate "machine learning" methods, from "statistical" methods ( Table 1: "Multivariable hierarchal logistic regression models*** (models which are based only on statistics - but there is no machine learning)", as an exclusion criterion ). This is clearly not the suitable platform to resolve this issue, but, the distinction between machine learning and statistics is not at all that clear. Specifically, under the term "supervised learning", any regression method (statistics) could be classified. So, logistic regression IS a machine learning method. So is LASSO and several other methods reported. Again, this is not the appropriate place for going into further details, but there is certainly some confusion, especially when in the results Logistic regression keeps appearing as a preferred method.

Again concerning terminology, the term "accuracy" appears often in the results section. Sometimes it is reported as a different outcome than i.e. ROC AUC, sensitivity and specificity. All the latter methods are quantifying "accuracy" in some way and some clarification is needed.

\section{Minor comments:}

Table 1: Treatment/Intervention, a parenthesis is missing.

Tables 7 \& 10: Maybe reverse the orientation of the column titles, it is impossible to read on a screen.

Are the rationale for, and objectives of, the Systematic Review clearly stated? Yes

Are sufficient details of the methods and analysis provided to allow replication by others? Yes

Is the statistical analysis and its interpretation appropriate?

Not applicable

Are the conclusions drawn adequately supported by the results presented in the review? Partly

Competing Interests: No competing interests were disclosed.

Reviewer Expertise: Statistics

I confirm that I have read this submission and believe that I have an appropriate level of expertise to confirm that it is of an acceptable scientific standard, however I have significant reservations, as outlined above. 
The benefits of publishing with F1000Research:

- Your article is published within days, with no editorial bias

- You can publish traditional articles, null/negative results, case reports, data notes and more

- The peer review process is transparent and collaborative

- Your article is indexed in PubMed after passing peer review

- Dedicated customer support at every stage

For pre-submission enquiries, contact research@f1000.com 